TRANSACTIONS OF THE

AMERICAN MATHEMATICAL SOCIETY

Volume 354, Number 1, Pages 151-178

S 0002-9947(01)02810-0

Article electronically published on July 13, 2001

\title{
WEAKLY DEFECTIVE VARIETIES
}

\author{
L. CHIANTINI AND C. CILIBERTO
}

\begin{abstract}
A projective variety $X$ is ' $k$-weakly defective' when its intersection with a general $(k+1)$-tangent hyperplane has no isolated singularities at the $k+1$ points of tangency. If $X$ is $k$-defective, i.e. if the $k$-secant variety of $X$ has dimension smaller than expected, then $X$ is also $k$-weakly defective. The converse does not hold in general. A classification of weakly defective varieties seems to be a basic step in the study of defective varieties of higher dimension. We start this classification here, describing all weakly defective irreducible surfaces. Our method also provides a new proof of the classical Terracini's classification of $k$-defective surfaces.
\end{abstract}

\section{INTRODUCTION}

In this paper we work over the complex field $\mathbf{C}$.

Let $X \subseteq \mathbf{P}^{r}$ be a reduced, irreducible (not necessarily smooth) projective variety of dimension $n$ and codimension $q=r-n$. Let $H$ be a hyperplane divisor on $X$ and let $\mathcal{H} \subseteq|H|$ be the (possibly incomplete) linear series cut out on $X$ by the hyperplanes of $\mathbf{P}^{r}$. We will assume that $X$ is non-degenerate, i.e. that $\mathcal{H}$ has dimension $r$, which is equivalent to saying that $X$ spans the whole of $\mathbf{P}^{r}$. Then, abusing notation, we will often identify the divisor $H$ with the unique hyperplane which cuts $H$ on $X$.

Now let $k$ be a non-negative integer and let $S^{k}(X)$ be the $k$-secant variety of $X$, i.e. the Zariski closure of the set

$$
\left\{P \in \mathbf{P}^{r}: P \text { lies in the span of } k+1 \text { independent points of } X\right\} \text {. }
$$

Of course $S^{0}(X)=X, S^{r}(X)=\mathbf{P}^{r}$ and $S^{k}(X)$ is empty if $k \geq r+1$. We will also set $S^{k}(X)=\emptyset$ if $k<0$. Notice that, for $k \geq-1$, one has

$$
s^{(k)}(X):=\operatorname{dim}\left(S^{k}(X)\right) \leq \min \{r, n(k+1)+k\} .
$$

The right hand side of (1.1) is called the expected dimension of $S^{k}(X)$ and will be denoted by $\sigma^{(k)}:=\sigma^{(k)}(X)$. We will also write $s^{(k)}$ instead of $s^{(k)}(X)$ if there is no danger of confusion. According to a definition given by Zak in [Zak], we will say that $X$ has a $k$-defect, or is $k$-defective, or is defective of index $k$ when strict inequality holds in (1.1). Notice that no variety is 0 -defective, and every variety is $k$-defective for $k \geq r+1$. Furthermore, if $X$ is $h$-defective for some $h \geq 1$, then it is also $k$-defective for all $k$ such that $s^{k}(X)<r$.

Recall now the classical lemma of Terracini (see Terr1] or, for modern versions, Adl], Dale1], Zak]), which says that, given a general point $P \in S^{k}(X)$, lying in the subspace $\left\langle P_{1}, \ldots, P_{k+1}\right\rangle$ spanned by $P_{1}, \ldots, P_{k+1}$ general points on $X$, then

Received by the editors March 1, 2000.

2000 Mathematics Subject Classification. Primary 14E25. 
the tangent space $T_{S^{k}(X), P}$ to $S^{k}(X)$ at $P$ is the span $T_{X, P_{1}, \ldots, P_{k+1}}$ of the tangent spaces $T_{X, P_{1}}, \ldots, T_{X, P_{k+1}}$, namely

$$
T_{S^{k}(X), P}=T_{X, P_{1}, \ldots, P_{k+1}}:=\left\langle T_{X, P_{1}}, \ldots, T_{X, P_{k+1}}\right\rangle .
$$

Therefore $X$ is $k$-defective if and only if:

$$
s^{(k)}=\operatorname{dim}\left(T_{X, P_{1}, \ldots, P_{k+1}}\right)<\sigma^{(k)}=\min \{r,(k+1)(n+1)-1\} .
$$

Now let $Y$ be a subscheme of a variety $X$ and let $\mathcal{L}$ be a linear system of Cartier divisors on $X$. We will denote by $\mathcal{L}(-Y)$ the linear system of all divisors in $\mathcal{L}$ containing $Y$. If $Y$ is a union of points $P_{1}, \ldots, P_{m}$, we write $\mathcal{L}\left(-P_{1} \cdots-P_{m}\right)$ for $\mathcal{L}(-Y)$. If $P_{1}, \ldots, P_{m}$ are smooth points of $X$, we let $\mathcal{L}\left(-2 P_{1} \cdots-2 P_{m}\right)$ be the linear system of all divisors in $\mathcal{L}\left(-P_{1} \cdots-P_{m}\right)$ having singular points at $P_{1}, \ldots, P_{m}$.

In case $\mathcal{L}=\mathcal{H}$ is, as above, the linear system of hyperplane divisors of $X$ in $\mathbf{P}^{r}$, then $\mathcal{H}\left(-P_{1} \cdots-P_{m}\right)$ is cut out on $X$ by the hyperplanes in $\mathbf{P}^{r}$ containing the subspace $\left\langle P_{1}, \ldots, P_{m}\right\rangle$, whereas $\mathcal{H}\left(-2 P_{1} \cdots-2 P_{m}\right)$ is cut out on $X$ by the hyperplanes in $\mathbf{P}^{r}$ containing the subspace $T_{X, P_{1}, \ldots, P_{m}}:=\left\langle T_{X, P_{1}}, \ldots, T_{X, P_{m}}\right\rangle$. These hyperplanes are called $m$-tangent hyperplanes to $X$. Hence (1.2) is equivalent to saying that $X$ is $k$-defective if and only if, for $P_{1}, \ldots, P_{k+1}$ general points on $X$, the system $\mathcal{H}\left(-2 P_{1} \cdots-2 P_{k+1}\right)$ is not empty and

$$
r-s^{(k)}-1=\operatorname{dim}\left(\mathcal{H}\left(-2 P_{1} \cdots-2 P_{k+1}\right)\right) \geq r-(k+1)(n+1) .
$$

One defines the $k$-defect of $X$ as the number

$$
\begin{aligned}
\delta_{k}(X) & =\operatorname{dim}\left(\mathcal{H}\left(-2 P_{1} \cdots-2 P_{k+1}\right)\right)-\max \{-1, r-(k+1)(n+1)\} \\
& =\sigma^{k}(X)-s^{k}(X),
\end{aligned}
$$

where $P_{1}, \ldots, P_{k+1}$ are general points on $X$. We will also use the shorter notation $\delta_{k}$ for $\delta_{k}(X)$ if there is no danger of confusion. Of course $X$ is $k$-defective if and only if $\delta_{k}>0$. $X$ is said to have a defect, or to be defective, if it has a non-zero $k$-defect for some $k$.

The first non-trivial example of a defective variety is the Veronese surface in $\mathbf{P}^{5}$. A general theory of defective varieties has been developed by several authors, both classical and modern (see e.g. [Pal1], [Terr2], [Sco1], [Br], [Zak], Dale2], Ad], [Fa], CJ1, etc.). We will not review here all known results in this theory, but only recall that, as we will also see later, there are no defective varieties of dimension 1. By contrast, as noticed by Palatini in $\mathrm{Pal2}$, there are defective surfaces, and he tried to classify them, but his classification contained some gaps. A complete classification, which fills up Palatini's gaps, is contained in Terracini's paper Terr2], and has been also worked out in modern times by Dale [Dale2] and Catalano-Johnson [CJ2], who apparently were both unaware of Terracini's paper.

In his paper [Terr2], Terracini implicitly used a crucial result which we now state and will prove in $\$ 2$ below. Notice that this result could be partly deduced from the results of Chapt. $\mathrm{V}$ of $[\mathrm{Zak}$, to which we refer for the general theory of defective varieties.

If $X$ is any variety as above, if $P_{1}, \ldots, P_{k+1} \in X$ are general points, and if $H \in \mathcal{H}\left(-2 P_{1}-\cdots-2 P_{k+1}\right)$ is a general hyperplane tangent at $P_{1}, \ldots, P_{k+1} \in X$, we can consider the contact variety of $H$, i.e. the union $\Sigma:=\Sigma_{P_{1}, \ldots, P_{k+1}}(H)$ of the irreducible components of $\operatorname{Sing}(H)$ containing $P_{1}, \ldots, P_{k+1}$. Since $P_{1}, \ldots, P_{k+1}$ are general points, an obvious monodromy argument shows that $\Sigma$ is equidimensional, and we denote by $\nu_{k}:=\nu_{k}(X)$ its dimension, which we will call the $k$-singular defect 
of $X$. Of course $\nu_{k} \leq n-1$, and we set $\nu_{k}=-1$ if $\mathcal{H}\left(-2 P_{1}-\cdots-2 P_{k+1}\right)$ is empty. We also set $h(\Sigma)=r-\operatorname{dim}(\mathcal{H}(-\Sigma))$. This is the number of conditions imposed by $\Sigma$ on the divisors of $\mathcal{H}$ containing it.

With all this in mind, we can state Terracini's theorem:

Theorem 1.1. Let $X \subset \mathbf{P}^{r}$ be a reduced, irreducible, non-degenerate, projective variety of dimension $n$. If $P_{1}, \ldots, P_{k+1} \in X$ are general points, and if $H \in$ $\mathcal{H}\left(-2 P_{1}-\cdots-2 P_{k+1}\right)$ is a general $(k+1)$-tangent hyperplane and $\Sigma$ is its contact variety of dimension $\nu_{k} \geq 0$, then

$$
k+1 \leq h(\Sigma) \leq(k+1)\left(1+\nu_{k}\right)-\delta_{k},
$$

and therefore

$$
(k+1) \nu_{k} \geq \delta_{k} .
$$

In particular, if $X$ is $k$-defective then $n-1 \geq \nu_{k}>0$, i.e. the general $(k+1)$ tangent hyperplane to $X$ has a contact variety of positive dimension.

This theorem explains the difficulty in finding varieties with large defect: as soon as $\delta_{k}$ increases, the variety $\Sigma$ imposes fewer and fewer conditions on the hyperplanes, so that it becomes more and more special.

Notice also that the converse to the final part of this statement is false in general: if $X$ is a cone, by imposing tangency at one point one gets tangency along a line, but on the other hand there are cones which are not defective at all. So one is led to the following definition:

Definition 1.2. $X$ is a $k$-weakly defective variety if the general $(k+1)$-tangent hyperplane to $X$ has a contact variety of positive dimension.

Notice that if $X$ is $h$-weakly defective for some $h \geq 1$, then it is also $k$-weakly defective for all $k \geq h$ such that there is a $(k+1)$-tangent hyperplane to $X$ at $k+1$ general points. The value of the singular defect $\nu_{k} \leq n-1$ plays an important role in the classifcation of weakly defective varieties. The maximal case $\nu_{k}=n-1$, which we will refer to as to the divisorial case, turns out to be simpler than the others. We will collect the main properties of weakly defective varieties in $\S 3$, and we will present a few basic examples in $\S 4$.

As we already pointed out, a $k$-defective variety is also $k$-weakly defective, the converse being in general false. However it turns out that, in order to understand defective varieties of a given dimension $n$, one first has to classify weakly defective varieties of any dimension smaller than $n$ (see [Sco1], [Terr2]). Unfortunately, such a classification problem, even for varieties of low dimension, seems to have never been really considered in the literature, except for:

(i) the case of 0 -weakly defective varieties, which are the varieties whose Gauss map is degenerate, whose classification is well known (see remark 3.1, ii, below); and

(ii) the case of 1-weakly defective surfaces, considered in the papers Sco2 and Terr2 by Scorza and Terracini.

The aim of this paper is to start filling up this gap, thus giving the full classification of weakly defective surfaces, which, of course, present the divisorial case. Our main result is the following theorem, proved in $\S 7$ :

Classification Theorem 1.3. Let $X \subset \mathbf{P}^{r}$ be a reduced, irreducible, non-degenerate, projective surface which is $k$-weakly defective. 
If $\delta_{k}>0$, i.e. if $X$ is $k$-defective, then $k \geq 1$ and either

(i) the contact curve of a general $k+1$-tangent hyperplane is irreducible, and then $r=3 k+2, \delta_{k}=1$ and $X$ is the 2 -Veronese embedding in $\mathbf{P}^{r}$ of a rational normal surface $Y$ of degree $k$ in $\mathbf{P}^{k+1}$; or,

(ii) the contact curve of a general $k+1$-tangent hyperplane is reducible, and then $X$ sits in an $(s+2)$-dimenional cone over a curve, with vertex a linear space of dimension $s \leq k-1$ and $r \geq 2 k+s+3$. The minimal such $s$ is characterized by the property that $X$ is $s$-defective but not $(s-1)$-defective, and one has $\delta_{k} \geq k-s$.

If $\delta_{k}=0$, i.e. if $X$ is not $k$-defective, then either

(iii) the contact curve of a general $k+1$-tangent hyperplane is irreducible, and then either $k=0$ and $X$ is the tangent developable to a curve, or

(iv) $r=9, k=2$ and $X$ is the 2 -Veronese embedding of a surface of degree $d \geq 3$ in $\mathbf{P}^{3}$, or

(v) $r=3 k+3$ and $X$ sits in the cone with vertex a point over a $k$-defective surface of type (i), or

(vi) $r=3 k+3$ and $X$ is the 2 -Veronese embedding in $\mathbf{P}^{r}$ of a surface $Y$ of degree $k+1$ in $\mathbf{P}^{k+1}$ with curve sections of arithmetic genus 1 , or

(vii) the contact curve of a general $k+1$-tangent hyperplane is reducible, and then $X$ sits in a $s+2$ dimensional cone over a curve, with vertex a linear space of dimension $s \leq k$ and $r \geq 2 k+s+3$. The minimal such $s$ is characterized by the property that $X$ is s-weakly defective but not $(s-1)$-defective.

The nature of the cases listed in the above statements and their properties are fully explained in $\S 4$.

Our approach to the proof is inspired by, but substantially simplifies, Terracini's original one. It is mainly based, as we said, on theorem 1.1, which we prove in $\S 2$ using some standard facts on deformations of divisors on a variety. These results from deformation theory also enable us to prove the following theorem, which, together with the classification theorem 1.3, should be seen as a wide extension of Arbarello-Cornalba's results in $\mathrm{ArCl}$ :

Theorem 1.4. Let $X \subset \mathbf{P}^{r}$ be a reduced, irreducible, non-degenerate, projective variety. Then the general point of every irreducible component of the contact variety of a general $(k+1)$-tangent hyperplane section $H$ is a double point for $H$. If, in addition, $X$ is not $k$-weakly defective for a given $k$ such that $r \geq(n+1)(k+1)$, then, given $P_{1}, \ldots, P_{k+1}$ general points on $X$, the general $(k+1)$-tangent hyperplane $H \in \mathcal{H}\left(-2 P_{1}-\cdots-2 P_{k+1}\right)$ is tangent to $X$ only at $P_{1}, \ldots, P_{k+1}$. Moreover such a hyperplane section $H$ has ordinary double points at $P_{1}, \ldots, P_{k+1}$.

For the proof of the classification theorem we also use a tool of independent interest, i.e. the classification, presented in $\S 5$, of certain families of divisors on a variety, classically called involutions. This classification extends the classical theorem of Castelnuovo and Humbert which classifies involutions on curves (see EnChi], Ma ). In addition, we use a basic lemma in Castelnuovo's theory, discussed in $\S 6$ (for a general reference on this latter subject we refer to [EiHa]), which enables us to determine some basic properties of the contact curve of a general $(k+1)$ tangent hyperplane to a $k$-weakly defective surface $X$.

As we mentioned already, we became aware of the importance of classifying weakly defective surfaces when we tried to understand Scorza's classification in Sco1] of 2-defective (not necessarily smooth) threefolds. In a future paper, with 
the aid of the present classification thoerem, we hope to come back to the subject of defective varieties of higher dimension.

\section{The infinitesimal Bertini theOREM, TERRACINI'S THEOREM, AND APPLICATIONS}

In this section, we prove Terracini's theorem 1.1. First we recall a few standard facts about deformation theory of divisors on a smooth variety.

Let $X$ be a smooth $n$-dimensional variety and let $H$ be an effective divisor on $X$. Then we have the normal bundle sequence

$$
0 \rightarrow T_{H} \rightarrow T_{X \mid H} \rightarrow N_{H, X} \simeq \mathcal{O}_{H}(H) \rightarrow T_{H}^{1} \rightarrow 0 .
$$

If we have a point $x \in H$, we let $z_{1}, \ldots, z_{n}$ be coordinates on $X$ centered at $x$, and we let $f\left(z_{1}, \ldots, z_{n}\right)=0$ be an equation of $H$ in this coordinate system. The Taylor expansion of $f$ at $x$ gives us

$$
f\left(z_{1}, \ldots, z_{n}\right)=\sum_{i=1}^{\infty} f_{i}\left(z_{1}, \ldots, z_{n}\right)
$$

where the $f_{i}$ 's are homogeneous polynomials of degree $i$. Then $H$ is singular at $x$ of multiplicity $m \geq 2$ if $f_{i} \equiv 0, i=1, \ldots, m-1$, but $f_{m}$ is not identically zero. In this case the tangent vector space $T_{X, x}$ to $X$ at $x$ coincides with the Zariski tangent space $T_{H, x}$ to $H$ at $x$. Moreover, $f_{m}\left(z_{1}, \ldots, z_{n}\right)=0$ is the equation of the tangent cone $\mathcal{T} C_{H, x}$ to $H$ at $x$, which sits in $T_{H, x}$ and does not depend on local coordinates.

Recall that $H$ is said to have an ordinary singularity of multiplicity $m$ at $x$ if $\mathcal{T} C_{H, x}$ is singular only at $x$. If $H$ has a double point at $x$, i.e. if $m=2$, then one says that the rank of this double point is $h$ if $\mathcal{T} C_{H, x}$ is a quadric of rank $h$. Then the vertex $\mathcal{A}_{H, x}$ of $\mathcal{T} C_{H, x}$ is a subspace of $T_{H, x}$ of dimension $n-h$ which is called the asymptotic space of $H$ at $x$.

The map $T_{X \mid H} \rightarrow N_{H, X} \simeq \mathcal{O}_{H}(H)$ appearing in (2.1) is locally given by sending $\frac{\partial}{\partial z_{i}} \rightarrow \frac{\partial f}{\partial z_{i} \mid H}$. Hence $T_{H}^{1}$ is supported at the scheme $S:=\operatorname{Sing}(H)$ defined by the vanishing of the derivatives of $f$, and $T_{H}^{1} \simeq \mathcal{O}_{S}(H)$.

Let us consider the natural restriction map

$$
\rho: H^{0}\left(H, N_{H, X}\right) \rightarrow H^{0}\left(S, T_{H}^{1}\right) \simeq H^{0}\left(S, \mathcal{O}_{S}(H)\right) .
$$

Giving a section $s \in H^{0}\left(H, N_{H, X}\right)$ is equivalent to giving a first order deformation of $H$, i.e. a flat family $\sigma: H_{\epsilon} \rightarrow \mathbf{D}$, where $\mathbf{D}$ is the spectrum of the ring $\mathbf{C}[\epsilon]$ of the dual numbers. Suppose we also have a first order deformation $\xi: x_{\epsilon} \rightarrow \mathbf{D}$ of $x$ in $X$ given by $z_{i}=\epsilon a_{i}, i=1, \ldots, n$. Then one has the following well known lemma, whose easy proof we reproduce here for convenience.

Lemma 2.1. Let us consider, as above, a smooth variety $X$, a point $x \in X$, an effective divisor $H$ on $X$ passing through $x$, a first order deformation $\sigma$ of $H$ in $X$ and a fist order deformation $\xi$ of $x$ in $X$. Then:

(i) If $x$ has multiplicity $m$ for $H$ and $\sigma$ has multiplicity at least $m-1$ along $\xi$, then $\rho(s)$ vanishes with order at least $m-1$ at $x$.

(ii) If $H$ has a double point of rank $h$ at $x$ and if $\sigma$ is singular along $\xi$, then for every tangent vector $v$ belonging to the asymptotic space $\mathcal{A}_{H, x}$, one has $v(\rho(s))=0$. Conversely if for every $v \in \mathcal{A}_{H, x}$ one has $v(\rho(s))=0$, then $\sigma$ is singular along a suitable first order deformation $\xi$ of $x$ in $X$. 
Proof. As above, let $z_{1}, \ldots, z_{n}$ be coordinates on $X$ centered at $x$, let $f\left(z_{1}, \ldots, z_{n}\right)$ $=0$ be the equation of $H$ in these coordinates and let $f\left(z_{1}, \ldots, z_{n}\right)+\epsilon g\left(z_{1}, \ldots, z_{n}\right)$ $=0$ be the equation of $\sigma$. The Taylor expansions of $f$ and $g$ give us

$$
\begin{aligned}
& f\left(z_{1}, \ldots, z_{n}\right)=\sum_{i=2}^{\infty} f_{i}\left(z_{1}, \ldots, z_{n}\right), \\
& g\left(z_{1}, \ldots, z_{n}\right)=\sum_{i=0}^{\infty} g_{i}\left(z_{1}, \ldots, z_{n}\right),
\end{aligned}
$$

where the $f_{i}$ 's and $g_{i}$ 's are homogeneous polynomials of degree $i$. The infinitesimal deformation $\xi$ of $x$ is given by $z_{i}=\epsilon a_{i}, i=1, \ldots, n$.

Notice that $\sigma$ has multiplicity at least $m-1$ along $\xi$ if and only if:

$$
D f\left(\epsilon a_{1}, \ldots, \epsilon a_{n}\right)+\epsilon D g\left(\epsilon a_{1}, \ldots, \epsilon a_{n}\right)=0,
$$

where $D$ is any partial derivative of order $j \leq m-2$. Since $f_{i} \equiv 0, i=0, \ldots, m-1$, we have $D f\left(\epsilon a_{1}, \ldots, \epsilon a_{n}\right)=0$, for any $D$ of order $j \leq m-2$. Thus (2.2) is equivalent to $\epsilon D g\left(\epsilon a_{1}, \ldots, \epsilon a_{n}\right)=0$ for any such $D$. This clearly implies that $g_{i} \equiv 0, i=1, \ldots, m-2$, proving (i).

In order to prove (ii), we may assume $f_{0}=f_{1} \equiv 0$ and $f_{2}\left(z_{1}, \ldots, z_{n}\right)=z_{1}^{2}+$ $\cdots+z_{h}^{2}$. Then $(2.2)$ is equivalent to $g_{0}=0$ and to the system of relations

$$
\begin{gathered}
2 a_{i}+\frac{\partial g_{1}}{\partial z_{i}}=0, \quad i=1, \ldots, h, \\
\frac{\partial g_{1}}{\partial z_{i}}=0, \quad i=h+1, \ldots, n .
\end{gathered}
$$

Since $\mathcal{A}_{H, x}$ has equations $z_{i}=0, i=1, \ldots, h$, the former set of relations tells us that for every tangent vector $v \in \mathcal{A}_{H, x}$, one has $v(\rho(s))=0$. Conversely, if such a condition is satisfied, then the second set of relations is satisfied, and from the first we can compute the $a_{i}$ 's, hence a first order deformation $\xi$ along which $\sigma$ stays singular.

As an immediate consequence, we have the following:

Infinitesimal Bertini's Theorem 2.2 . Let $X$ be a smooth irreducible variety and let $\left\{H_{y}\right\}_{y \in Y}$ be an algebraic family of Cartier divisors on $X$ parametrized by a reduced, irreducible variety $Y$. Let $y \in Y$ be a general point, let $S:=S_{y}$ be the singular locus of $H:=H_{y}$. Let $v$ be any tangent vector to $Y$ at $y$ and let $s \in$ $H^{0}\left(H, N_{H, X}\right)$ be the section of the normal bundle $N_{H, X}$ of $H$ in $X$ corresponding to the first order deformation $\sigma$ of $H$ determined by $v$. Then $s$ vanishes along $S$.

In particular, if $Y$ is contained in a linear system $\mathcal{L}$, then the projective tangent space to $Y$ at $y$ in $\mathcal{L}$ is contained in $\mathcal{L}(-S)$.

Proof. Since $y \in Y$ is general, if $x \in S$ is any singular point of $H$, there is a first order deformation of $x$ along which $\sigma$ stays singular. Then one applies the previous lemma.

Now we give a proof of Terracini's theorem 1.1, which essentially reproduces the proof given in $\mathrm{CiHi}$.

Proof of theorem 1.1. Let $\mathcal{X}^{k+1}$ be the open subset of $X^{k+1}$ described by $(k+1)$ tuples formed by distinct points. Given an element $D \in \mathcal{X}^{k+1}$, we will abuse 
notation and denote by $D$ also the subset of $X$ formed by the $k+1$ points of $D$. Consider now the closure $I$ in $\mathcal{H} \times X^{k+1}$ of the incidence correspondence

$$
\left\{(H, D) \in \mathcal{H} \times \mathcal{X}^{k+1}: D \subseteq \operatorname{Sing}(H)\right\},
$$

and let $p_{1}: I \rightarrow \mathcal{H}$ and $p_{2}: I \rightarrow X^{k+1}$ be the two projections.

Since $\nu_{k} \geq 0$, the map $p_{2}$ is dominant and its general fiber is a projective space of dimension $l=r-(k+1)(n+1)+\delta_{k}$. Then there is only one irreducible component $J$ of $I$ which dominates $X^{k+1}$, and one has

$$
\operatorname{dim}(J)=l+n(k+1)=r-k-1+\delta_{k} .
$$

Now consider the family of divisors on $X$ given by $Y=p_{1}(J) \subseteq \mathcal{H}$. If $(H, D) \in J$ is general, with $D=\left(P_{1}, \ldots, P_{k+1}\right)$, then every component of $\Sigma=\Sigma_{P_{1}, \ldots, P_{k+1}}(H)$ intersects the set of smooth points of $X$. Hence we may apply theorem 2.2 and conclude that the projective tangent space to $Y$ at $H$ is contained in $\mathcal{H}(-\Sigma) \subseteq$ $\mathcal{H}(-D)$. Since $D$ is formed by $k+1$ general points of $X$, then $\operatorname{dim}(\mathcal{H}(-D))=$ $r-k-1$, and hence

$$
\operatorname{dim}(Y) \leq \operatorname{dim}(\mathcal{H}(-\Sigma)) \leq \operatorname{dim}(\mathcal{H}(-D))=r-k-1
$$

Finally, by the very meaning of the singular defect $\nu_{k}$, we see that the general fibre of the restriction of $p_{1}$ to $J$ has dimension $(k+1) \nu_{k}$. In conclusion we have:

$$
r-k-1+\delta_{k}=\operatorname{dim}(J)=\operatorname{dim}(Y)+(k+1) \nu_{k}
$$

which, together with (2.3), yields the assertion.

Notice that the classical theorem of Bertini, which says that the singular points of a general member of a linear system are base points of the system itself, is an obvious consequence of theorem 2.2, on which we will not dwell here. Rather, we want to point out the following interesting consequence of theorem 2.2:

Proposition 2.3. Let $X$ be a smooth variety and let $\mathcal{L}$ be a linear system of divisors on $X$. Let $P$ be a general point of $X$, let $m$ be a positive integer and let $\mathcal{L}(-m P)$ be the sublinear system of $\mathcal{L}$ formed by all divisors with a point of multiplicity at least $m$ at $P$. If $\mathcal{L}(-m P)$ is not empty, then the general element $H \in \mathcal{L}(-m P)$ has a point of multiplicity exactly $m$ at $P$.

Proof. The assertion is trivial for $m=1$, so we assume $m \geq 2$. Consider the incidence correspondence $M=\{(P, H) \in X \times \mathcal{L}: H \in \mathcal{L}(-m P)\}$. There is a unique irreducible component $N$ of $M$ which dominates $X$ under the projection to the first factor. The image $Y$ of $N$ in $\mathcal{L}$ under the projection to the second factor parametrizes a family of divisors in $\mathcal{L}$ which is not, by Bertini's theorem, a linear system, since the singularities of the general member of this family describe the whole of $X$. Suppose for a contradiction that $\mathcal{L}(-m P)=\mathcal{L}(-(m+1) P)$. Then by lemma 2.1 we see that if $y \in Y$ is a general point corresponding to a divisor $H \in \mathcal{L}(-m P)$, if $v$ is any tangent vector to $Y$ at $y$, and if $s \in H^{0}\left(H, N_{H, X}\right)$ is the section of the normal bundle $N_{H, X}$ corresponding to the first order deformation of $H$ determined by $v$, then $s$ vanishes at $P$ with multiplicity at least $m$. This implies that the projective tangent space to $Y$ at $y$ in $\mathcal{L}$ is contained in $\mathcal{L}(-m P)$, and thus $\operatorname{dim}(Y) \leq \operatorname{dim}(\mathcal{L}(-m P))$. On the other hand we know that $\operatorname{dim}(\mathcal{L}(-m P))<$ $\operatorname{dim}(Y)$, since $\mathcal{L}(-m P)$ is a closed subset of $Y$; but it is not equal to $Y$ because $Y$ is not a linear system. Thus we have reached a contradiction. 
Remark 2.4. Proposition 2.3 could be easily extended to a more general situation, if we replace the point $P$ by a subvariety $Z$ of $X$ which is allowed to vary in such a way as to describe the whole of $X$. We think this extension is obvious and therefore we do not insist on this here.

From proposition 2.3 one immediately deduces

Corollary 2.5. Let $X \subset \mathbf{P}^{r}$ be a smooth variety and let $\mathcal{H}$ be the linear system of hyperplane sections of $X$. If $P_{1}, \ldots, P_{k+1}$ are general points of $X$, if $\mathcal{H}\left(-2 P_{1}-\cdots-2 P_{k+1}\right)$ is not empty, and if $H$ is a general divisor of this linear system, then the general point of each component of $\Sigma_{P_{1}, \ldots, P_{k+1}}(H)$ is a double points for $H$.

This corollary proves the first part of theorem 1.4 stated in the introduction. Before giving the proof of its second part, we recall the following well known fact:

Proposition 2.6. Let $X \subseteq \mathbf{P}^{r}$ be an irreducible, reduced, non-degenerate projective variety of dimension $n$ and let $k$ be a non-negative integer such that $k<r-n$. Let $P_{1}, \ldots, P_{k+1}$ be general points of $X$. Then the schematic intersection of $X$ with the subspace $\left\langle P_{1}, \ldots, P_{k+1}\right\rangle$ is the union of the points $P_{1}, \ldots, P_{k+1}$.

Proof. By taking the section of $X$ with a general subspace of codimension $n-1$, it suffices to prove the assertion only for curves. Then, by taking the projection of the curve $X$ from a general point $P \in X$, it suffices to prove the assertion for $k=1$, in which case it is true, a result known "since forever" and recently called the trisecant lemma (see for example [ChCi]).

Now we are ready to finish the

Proof of theorem 1.4. We will prove the final assertion in the theorem in the case when $X$ is smooth. Otherwise one can easily adapt the argument by passing to a resolution of the singularities. We leave these details to the reader.

Let us go back to the proof of theorem 1.1, from which we keep the notation. We thus consider the variety $Y \subset \mathcal{H}$ of codimenision $k+1$ in $\mathcal{H}$. Let $H \in Y$ be a general point. Then $H$ is tangent to $X$ at $k+1$ general points $P_{1}, \ldots, P_{k+1}$. Since $X$ is not $k$-weakly defective, $H$ has isolated singularities at $P_{1}, \ldots, P_{k+1}$. Then the infinitesimal Bertini theorem 2.2 tells us that $T_{Y, H}$ is contained in $\mathcal{H}\left(-P_{1}-\cdots-P_{k+1}\right)$. Since the two spaces have the same dimension, they coincide. On the other hand $\mathcal{H}\left(-P_{1}-\cdots-P_{k+1}\right)$ is cut out on $X$ by the hyperplanes through $\left\langle P_{1}, \ldots, P_{k+1}\right\rangle$. Then proposition 2.6 and the infinitesimal Bertini theorem 2.2 again forbid the presence of singularities for $H$ other than $P_{1}, \ldots, P_{k+1}$. Furthermore, proposition 2.6 and lemma 2.1,ii also forbid the presence of non-ordinary singularities at $P_{1}, \ldots, P_{k+1}$.

\section{WEAKLY DEFECTIVE VARIETIES: MAIN PROPERTIES}

In this section we collect a few general remarks and propositions on weakly defective and defective varieties, which are either immediate consequences of the definition or easy consequences of theorem 1.1. Unless otherwise stated, $X \subseteq$ $\mathbf{P}^{r}$ will be an irreducible, reduced $n$-dimensional projective variety, and $k$ a nonnegative integer. We start with the following: 
Remark 3.1. (i) There are no weakly defective curves. Actually, by theorem 1.1 there are also no defective curves.

(ii) $X$ is 0 -weakly defective if and only if its Gauss map

$$
\gamma_{X}: P \in X-\operatorname{Sing}(X) \rightarrow T_{X, P} \in \operatorname{Grass}(n, r)
$$

is degenerate, i.e. it is not generically finite. These varieties are singular, since a theorem of F. Zak ensures that the Gauss map is finite for a smooth variety (see Zak $)$. The classification of these varieties is classical; it goes back to C. Segre ( Segre] ) and is also contained in $\mathrm{GrHa}$, §2. It turns out that the closure of the general fibre of $\gamma_{X}$ is a projective space; hence $X$ is a developable scroll, i.e. a scroll such that along the general generating space, the tangent space to $X$ is constant.

In the case of a surface $X$, this follows easily from theorem 1.1. In fact the general tangent hyperplane to $X$ is tangent to $X$ along a curve $\Sigma$ which, according to theorem 1.1, imposes 2 conditions on the hyperplanes which have to contain it. Hence $\Sigma$ is a line, and $S$ is a scroll, which is developable. As we know, developable surfaces are either cones or tangent surfaces to curves (see [Segre, GrHa]).

Given all this, from now on we will consider $k$-weakly defective varieties for $k \geq 1$.

(iii) If $X$ is a weakly defective variety of index $k-1$ and if $\nu_{k} \geq 0$, i.e. if the linear system $\mathcal{H}\left(-2 P_{1}-\cdots-2 P_{k+1}\right)$ is non-empty for general points $P_{1}, \ldots, P_{k+1}$, then $X$ is also weakly defective of index $k$.

(iv) If $r \leq(k+1)(n+1)-1$, i.e. if $\sigma^{(k)}=r$, and $\nu_{k} \geq 0$, then the linear system $\mathcal{H}\left(-2 P_{1}-\cdots-2 P_{k+1}\right)$ is non-empty for general points $P_{1}, \ldots, P_{k+1}$. Hence $X$ is actually $k$-defective and therefore also $k$-weakly defective.

(v) If the general projection $X^{\prime}$ of $X$ in $\mathbf{P}^{r-1}$ is a weakly defective variety of index $k$, then $X$ is also a weakly defective variety of index $k$. The converse holds provided $\nu_{k}\left(X^{\prime}\right) \geq 0$, which is certainly the case whenever $r \geq(n+1)(k+1)$, i.e. whenever $\sigma^{(k)}<r$.

(vi) If the hyperplane section of $X$ is a weakly defective variety of index $k$ and if $\nu_{k}(X) \geq 0$, then $X$ itself is a weakly defective variety of index $k$. If $\nu_{k}(X) \geq 2$, the converse also holds.

A bound on the index of a $k$-weakly defective variety $X$ in $\mathbf{P}^{r}$, in terms of its codimension $q$, is given by the following proposition. This bound is $\operatorname{sharp}$ for $k=1$, but for higher values of $k$ it could be improved with the same ideas contained in the proof below. We do not dwell on this here.

Proposition 3.2. If $k \geq 1$ and $\nu_{k} \geq 0$, then $k \leq r-n-1=q-1$. In addition, if $q \geq 2$ then $k \leq q-2$.

Proof. If $P_{1}, \ldots, P_{q} \in X$ are general points, then there is only one hyperplane $H$ which is tangent to $X$ at $P_{1}$ and passes through $P_{2}, \ldots, P_{q}$. This immediately shows that $k+1 \leq q$, since $H$ cannot contain another general point of $X$. Let us exclude the case $q=k+1 \geq 2$. In this case $H$, which is tangent to $X$ at $P_{1}$, should also be tangent to $X$ at $P_{2}, \ldots, P_{q}$. In particular, every element in the pencil of hyperplane sections $\mathcal{H}\left(-2 P_{1}-P_{2}-\cdots-P_{q-1}\right)=\mathcal{H}\left(-2 P_{1}-\cdots-2 P_{q-1}\right)$ would be everywhere non-reduced, contrary to Bertini's theorem.

Next we discuss projections from tangent spaces and relations with (weak) defectiveness. First of all, let us introduce some more definitions and notation. Let $X \subseteq \mathbf{P}^{r}$ be, as above, a variety of dimension $n$ and let $P_{1}, \ldots, P_{k}$ be general points 
of $X$. Consider the subspace $T_{X, P_{1}, \ldots, P_{k}}=\left\langle T_{X, P_{1}}, \ldots, T_{X, P_{k}}\right\rangle$, which has dimension $s^{(k-1)}$ by (1.2). Consider the projection $\tau_{X, k}:=\tau_{X, P_{1}, \ldots, P_{k}}$ of $\mathbf{P}^{r}$ with centre $T_{X, P_{1}, \ldots, P_{k}}$. We will call such a projection a general $k$-tangential projection of $X$. Abusing notation, we will often denote by $\tau_{X, k}:=\tau_{X, P_{1}, \ldots, P_{k}}$ also the restriction of this projection to $X$. We will write $\tau_{X}$ instead of $\tau_{X, 1}$, a map which we will call a general tangential projection of $X$.

We will set $X_{k}:=\tau_{X, k}(X), n_{k}:=n_{k}(X)=\operatorname{dim}\left(X_{k}\right)$ and $\mu_{k}:=\mu_{k}(X)=n-n_{k}$, so that $\mu_{k}$ is the dimension of the general fibre of $\tau_{X, k}: X \rightarrow X_{k}$. We will set $\tau_{X, 0}=\mathrm{id}$, and accordingly $n_{0}=n$ and $\mu_{0}=0$.

Lemma 3.3. Let $X \subseteq \mathbf{P}^{r}$ be a reduced, irreducible, non-degenerate projective variety of dimension $n$. For every positive integer $k \leq r-1$ one has:

(i) $n_{k} \leq n_{k-1}$ or, equivalently, $\mu_{k-1} \leq \mu_{k}$;

(ii) $n_{k}=s^{(k)}-s^{(k-1)}-1$;

(iii) $\nu_{k} \geq \mu_{k}$;

(iv) $s^{(k)}=k+\sum_{i=0}^{k} n_{k}=(k+1)(n+1)-1-\sum_{i=0}^{k} \mu_{j}$, and hence $\delta_{k} \leq \sum_{i=0}^{k} \mu_{j}$, with the equality holding if and only if $r \geq(k+1)(n+1)-1$, i.e. if $r \geq \sigma^{(k)}$.

Proof. Notice that $\tau_{X, k}=\tau_{X_{k-1}, 1} \circ \tau_{X, k-1}$. This immediately yields (i).

A general tangent space to $X_{k}$ is the projection from $T_{X, P_{1}, \ldots, P_{k}}$ of the tangent space $T_{X, P_{k+1}}$ to $X$ at a general point $P_{k+1}$. Hence it has dimension $s^{(k)}-s^{(k-1)}-1$. This proves (ii).

A general hyperplane section $H$ through $T_{X, P_{1}, \ldots, P_{k+1}}$ is the pull-back, via $\tau_{X, k}$, of a general tangent hyperplane section $H^{\prime}$ to $X_{k}$ at $\tau_{X, k}\left(P_{k+1}\right)$. Since $H^{\prime}$ is certainly singular at $\tau_{X, k}\left(P_{k+1}\right)$, then $H$ is singular along $\tau_{X, k}^{-1}\left(\tau_{X, k}\left(P_{k+1}\right)\right)$, which is a $\mu_{k^{-}}$ dimensional variety containing $P_{k+1}$. The assertion follows by the definition of the singular defect. This proves (iii).

Part (iv) follows by summing the relations $n_{i}=s^{(i)}-s^{(i-1)}-1, i=1, \ldots, k$, that we get from (ii).

Remark 3.4. From lemma 3.3 we deduce that $\delta_{k} \leq(k+1) \mu_{k} \leq(k+1) \nu_{k}$, which gives another proof of a part of theorem 1.1.

Proposition 3.5. Let $X \subseteq \mathbf{P}^{r}$ be a reduced, irreducible, non-degenerate, projective variety of dimension $n$.

(i) If $X$ is $k$-defective, then $\mu_{k}>0$; that is, a general $k$-tangential projection $\tau_{X, k}: X \rightarrow X_{k}$ has positive dimensional fibres.

(ii) Conversely, if $r-s^{(k-1)}-1 \geq n$, which is the case if $r \geq(k+1)(n+1)-1$, i.e. if $r \geq \sigma^{(k)}$, and if a general $k$-tangential projection $\tau_{X, k}: X \rightarrow X_{k}$ has positive dimensional fibres, then $X$ is $k$-defective.

Proof. Suppose $X$ is $k$-defective and assume $\mu_{k}=0$. Then lemma 3.3, i, iv would give $s^{(k)}=(k+1)(n+1)-1 \geq \sigma^{(k)}$, and so equality would hold, a contradiction. This proves (i).

Let us prove (ii). Since $n_{k}<n$, by lemma 3.3, ii and the hypothesis, $s^{(k)}<r$. On the other hand, we have

$$
s^{(k)}<s^{(k-1)}+n+1 \leq k(n+1)+n=(k+1)(n+1)-1,
$$

which proves that $s^{(k)}<\sigma^{(k)}$, i.e. that $X$ is $k$-defective. 
Proposition 3.6. Let $X \subseteq \mathbf{P}^{r}$ be a reduced, irreducible, non-degenerate, projective variety of dimension $n$, let $h \geq 0$ be an integer, and suppose $X$ is not $(h-1)$ defective and $r-s^{(h-1)}-1 \geq n$, which is the same as saying that $r \geq(h+1)(n+1)-1$, i.e. $r \geq \sigma^{(h)}$. Then $X$ is $k$-weakly defective for some $k \geq h$ if and only if $X_{h}$ is $(k-h)$-weakly defective.

Proof. The assertion is trivially true for $k=0$. So we can proceed by induction on $k$. In order to finish the proof, it therefore suffices to prove the assertion only for $h=1$, where we have a generically finite map $\tau_{X}: X \rightarrow X_{1} \subset \mathbf{P}^{q-1}$. Let $P_{1}, \ldots, P_{k+1}$ be general points of $X$. The hyperplanes tangent to $X_{1}$ at the general points $\tau_{X}\left(P_{2}\right), \ldots, \tau_{X}\left(P_{k+1}\right)$ are cut out on $\mathbf{P}^{q-1}$ by hyperplanes in $\mathbf{P}^{r}$ containing $T_{X, P_{1}}$ and tangent to $X$ at $P_{2}, \ldots, P_{k+1}$, i.e. by hyperplanes tangent to $X$ at $P_{1}, \ldots, P_{k+1}$. Since $\tau_{X}$ is generically finite, so is its restriction to such general tangent hyperplane sections, and, by the very meaning of the singular defect, we see that $\nu_{k}(X)=\nu_{k-1}\left(X_{1}\right)$, proving the assertion.

\section{EXAmples}

We collect in this section some basic examples. Among them are all the ones listed in the classification theorem 1.3.

Before starting, we point out the following elementary, but useful, criterion which tells us when we have a variety sitting in a cone (for further information, see Ga]).

Proposition 4.1. Let $X \subset \mathbf{P}^{r}$ be an irreducible, reduced, projective variety of dimension $n$. Then $X$ is contained in an $(s+m+1)$-dimensional cone over a reduced, irreducible variety $Y$ of dimension $m<n$, with vertex a linear space $V$ of dimension $s$, if and only if the general tangent space to $X$ intersects $V$ along a subspace of dimension $n-m-1$.

Proof. It suffices to remark that $X$ sits in such a cone if and only if the projection of $X$ from $V$ has dimension $m$. Hence such a projection generically has rank $m$ on $X$, which is equivalent to its having $m$ on the general tangent space to $X$. This happens if and only if such a space meets $V$ along a subspace of dimension $n-m-1$.

We stress the following obvious consequence:

Corollary 4.2. Let $X \subset \mathbf{P}^{r}$ be an irreducible, reduced, non-degenerate, projective variety of dimension $n$. Then there is no subspace $V$ of dimension $s \leq r-2$ such that a general tangent space to $X$ meets $V$ along a subspace of dimension $n-1$.

Proof. Otherwise the projection of $X$ from $V$ to $\mathbf{P}^{r-s-1}$ would be a point, a contradiction to the non-degeneracy, since $r-s-1 \geq 1$.

Now we come to our examples.

Example 4.3. Any non-degenerate variety $X \subset \mathbf{P}^{r}$ of dimension $n$ contained in an $(s+m+1)$-dimensional cone $W$ over a reduced, irreducible variety $Y$ of dimension $m<n$, with vertex a linear space $V$ of dimension $s$, is $k$-defective whenever $s+2 \leq$ $(k+1)(n-m)$ and $r \geq(m+1)(k+1)+s+1$.

Indeed, according to proposition 4.1, the general tangent space to $X$ cuts $V$ in a subspace of dimension $n-m-1$. Since $s+2 \leq(k+1)(n-m)$, then $k+1$ such tangent spaces span a subspace of dimension at most $s+(k+1)(m+1)$, and since $r \geq(m+1)(k+1)+s+1$, this is a proper subspace of $\mathbf{P}^{r}$. On the other hand, we 
also have $s+(k+1)(m+1) \leq(k+1)(n+1)-2$, and hence (1.2) implies that $X$ is $k$-defective.

Notice that the above considerations show that $\delta_{k} \geq(n-m)(k+1)-s-1$, so that the $k$-defect $\delta_{k}$ can become arbitrarily large in these examples.

Notice also that, if $P_{1}, \ldots, P_{k+1}$ are general points of $X$, the space $T_{X, P_{1}, \ldots, P_{k+1}}$ is contained in $T_{W, P_{1}, \ldots, P_{k+1}}$, a space which is tangent to $W$ along the rulings $\Pi_{1}, \ldots, \Pi_{k+1}$ passing through $P_{1}, \ldots, P_{k+1}$. Then $T_{W, P_{1}, \ldots, P_{k+1}}$ is also tangent to $X$ along the subvarieties $X_{1}, \ldots, X_{k+1}$ cut out by $\Pi_{1}, \ldots, \Pi_{k+1}$ on $X$. Notice that the $X_{i}$ 's are fibres of the projection $\pi: X \rightarrow Y$ from $V$; hence they have positive dimension $n-m$. So we see that $\nu_{k} \geq n-m>0$, and that the contact variety is reducible in this case.

Similarly, if, in the above situation, one has $s+1 \leq(k+1)(n-m)$ and $r \geq$ $(m+1)(k+1)+s+1$, then $X$ is $k$-weakly defective. In fact, by following the same argument as before, we see that either $X$ is $k$-defective, or $T_{X, P_{1}, \ldots, P_{k+1}}$ has exactly dimension $s+(k+1)(m+1)$ and coincides with $T_{W, P_{1}, \ldots, P_{k+1}}$. Then we conclude, arguing as above, that $X$ is $k$-weakly defective.

We notice that there are examples of varieties $X$ as above which are smooth. By way of example, consider the following situation. Let $Y$ be a smooth, irreducible, non-degenerate $m$-dimensional variety and let $Z=\mathbf{P}(\mathcal{E})$ be a $\mathbf{P}^{k}$-bundle over $Y$, where $\mathcal{E}$ is a vector bundle of rank $k+1$ on $Y$. We assume that $\mathcal{O}_{Z}(1)$ is very ample and embeds $Z$ in a $\mathbf{P}^{s}$. Let $\mathcal{L}$ be a very ample line bundle on $Y$ which embeds $Y$ in a $\mathbf{P}^{h}$. The variety $X=\mathbf{P}(\mathcal{E} \oplus \mathcal{L})$ is smooth, of dimension $n=k+m+1$. We also assume that $\mathcal{O}_{X}(1)$ is very ample and embeds $X$ in a $\mathbf{P}^{r}$, in which we have a subspace $V$ of dimension $s$ where $Z$ sits and a subspace $U$ of dimension $h$ where $Y$ sits, and $V$ and $U$ span all of $\mathbf{P}^{s}$, so that $r=r+h+1$. The projective construction of $X$ is well known: a generating $\mathbf{P}^{k+1}$ of $X$ is obtained by joining a point $P$ of $Y$ in $U$ with the generating $\mathbf{P}^{k}$ of $Z$ corresponding to $P$ in $V$. Hence $X$ also lies in the cone over $Y$ with vertex $V$.

For a different example which is not a scroll, take a smooth, irreducible, nondegenerate rational curve $C \subset \mathbf{P}^{h}$, and take the cone $W$ over $C$ with vertex a general line $V$ in $\mathbf{P}^{r}, r=h+2$, so that $W$ is smooth off $V$. The group of Weil divisors of $W$, modulo linear equivalence, is generated by $H$, the hyperplane class, and $\Pi$, the class of a ruling. It is not difficult to verify (see for example the argument in DiG, §3) that the general element $X$ in a linear system of the form $h H+\Pi, h \geq 1$, is smooth, irreducible and non-degenerate. For $h=1$ we find examples essentially the same as the previous ones, whereas for $h>1$ we find different examples of 2 -defective and 1-weakly defective surfaces. Notice that the assumption about the rationality of $C$ is not really necessary in order to perform the above construction.

Example 4.4. Let $Y$ in $\mathbf{P}^{s}$ be a $k$-defective, irreducible, reduced and non-degenerate variety of dimension $n$, with $k \geq 1$ and $s \geq(k+1)(n+1)-1$; let $X$ be any irreducible, reduced, non-degenerate, $n$-dimensional variety in $\mathbf{P}^{r}, r=s+1$, contained in the cone $W$ over $Y$ with vertex at a point $V$. Then $X$ is $k$-weakly defective.

One can see this in the following way. Let $P$ be a general point of $X$. If the tangent space $T_{X, P}$ contains the point $V$, then proposition 4.1 says that $X$ is a cone and $X$ is 0 -weakly defective, hence also $k$-weakly defective for any $k \geq 0$. Otherwise let us consider the tangential projection $\tau_{X, P}: X \subseteq \mathbf{P}^{r} \rightarrow X_{1} \subseteq \mathbf{P}^{r-n-1}$. We set $V^{\prime}=\tau_{X, P}(V) \in \mathbf{P}^{r-n-1}$, and also consider the projection $\sigma: \mathbf{P}^{r-n-1} \rightarrow \mathbf{P}^{r-n-2}$ 
from $V^{\prime}$. Notice that $\left\langle V, T_{X, P}\right\rangle=T_{W, P}$, so that the composite projection $\sigma \circ \tau$ : $\mathbf{P}^{r} \rightarrow \mathbf{P}^{r-n-2}$ is nothing but the tangential projection $\tau_{W, P}$.

We denote by $U$ the hyperplane in $\mathbf{P}^{r}$ in which $Y$ sits. We may actually assume that $U$ is a general hyperplane containing $P$. We can then consider also the tangential projection $\tau_{Y, P}: U \rightarrow \mathbf{P}^{r-n-2}$. Since $T_{W, P}=\left\langle V, T_{X, P}\right\rangle=\left\langle V, T_{Y, P}\right\rangle$, we see that $T_{Y, P}=T_{W, P} \cap U$, so that $\tau_{Y, P}$ is the restriction of $\tau_{W, P}$ to $U$.

By the above considerations, we see that the image $W_{1}$ of $W$ via $\tau_{X, P}$ is the cone with vertex $V^{\prime}$ over the variety $Y_{1} \subset \mathbf{P}^{r-n-2}$ which is the projection of $W$ via $\tau_{W, P}$. Since all the generating lines of $W$ are contracted by $\tau_{W, P}$, we see that this is the same as the image via $\tau_{Y, P}$ of $Y$.

Now, if $\operatorname{dim}\left(Y_{1}\right)<\operatorname{dim}(Y)$, which is certainly the case if $k=1$ by proposition 3.5 , i, then

$$
\operatorname{dim}\left(W_{1}\right)=\operatorname{dim}\left(Y_{1}\right)+1<\operatorname{dim}(Y)+1=n+1 .
$$

Hence, either also $\operatorname{dim}\left(X_{1}\right)<\operatorname{dim}(X)$ and therefore $X$ is 1-defective by proposition 3.5 , ii, or

$$
\operatorname{dim}\left(X_{1}\right)=\operatorname{dim}(X)=n \leq \operatorname{dim}\left(W_{1}\right)<n+1,
$$

which proves that $X_{1}=W_{1}$. Thus $X_{1}$ is a cone; hence it is 0 -defective. Then $X$ is 1-defective, and therefore also $k$-defective, by proposition 3.6.

The assertion is thus proved if either $k=1$ or $\operatorname{dim}\left(Y_{1}\right)<\operatorname{dim}(Y)$. If $k>1$ and $\operatorname{dim}\left(Y_{1}\right)=\operatorname{dim}(Y)$, then $Y_{1}$ is $(k-1)$-defective by proposition 3.6 and $X_{1}$ sits in the cone over $Y_{1}$ from $V^{\prime}$. We may then apply induction to $X_{1}$ and assert that it is $(k-1)$-weakly defective. Then propostion 3.6 again ensures that $X$ is $k$-weakly defective, as desired.

Example 4.5. Let $Y$ be a reduced, irreducible variety of dimension $n \geq 2$ in $\mathbf{P}^{s}$, of minimal degree $s-n+1$. Notice that we allow $n=s$, in which case $Y$ coincides with $\mathbf{P}^{s}$. The classification of these varieties is well known; see [Ha]. They are projectively Cohen-Macaulay, so that, in particular, the quadrics in $\mathbf{P}^{s}$ cut out on them a complete linear system, which turns out to be of dimension

$$
r=s(n+1)-\frac{(n-2)(n+1)}{2}-1
$$

(see either the proof of proposition 3.23 from EiHa or proposition 6.1 below). Consider the 2-Veronese embedding $X$ of $Y$ in $\mathbf{P}^{r}$. We claim that $X$ is $k$-defective for every $k$ such that

$$
s-\left[\frac{n-2}{2}\right]-1 \leq k \leq s-1 .
$$

Indeed, for every such value of $k$ one has

$$
r-(n+1)(k+1) \leq r-(n+1)\left(s-\left[\frac{n-2}{2}\right]\right) \leq r-(n+1)\left(s-\frac{n-2}{2}\right)=-1 .
$$

On the other hand, we claim that for general points $P_{1}, \ldots, P_{k+1}$ on $X$, the linear system $\mathcal{H}\left(-2 P_{1}-\cdots-2 P_{k+1}\right)$ is not empty. In fact, let $Q_{1}, \ldots, Q_{k+1}$ be the corresponding points on $Y$. The system certainly contains the Veronese image of the linear system cut out on $Y$ by the quadric cones in $\mathbf{P}^{s}$ with vertex at $\left\langle Q_{1}, \ldots, Q_{k+1}\right\rangle$. Since $s-k-1<n$ in this case, we see that the general projection of $Y$ from $\left\langle Q_{1}, \ldots, Q_{k+1}\right\rangle$ surjects onto $\mathbf{P}^{s-k-1}$. Hence there is no quadric cone 
with vertex at $\left\langle Q_{1}, \ldots, Q_{k+1}\right\rangle$ containing $Y$, and therefore the system in question is not empty of dimension $\left(\begin{array}{c}s-k+1 \\ 2\end{array}\right)-1$. This proves that actually

$$
\begin{aligned}
\delta_{k}(X) & =\operatorname{dim}\left(\mathcal{H}\left(-2 P_{1}-\cdots-2 P_{k+1}\right)\right)-\max \{-1, r-(n+1)(k+1)\} \\
& \geq\left(\begin{array}{c}
s-k+1 \\
2
\end{array}\right)
\end{aligned}
$$

In the surface case $n=2$, one has $k=s-1$. The surface $X$ is thus $s$-defective. We claim that $\delta_{k}=1$ in this case. In fact, if we take $P_{1}, \ldots, P_{s}$ general points on $X$, the general tangent hyperplane at $P_{1}, \ldots, P_{s}$ is tangent along a curve $\Sigma$. Let $Q_{1}, \ldots, Q_{s}$ be the corresponding points on $Y$ and let $\Gamma$ be the curve on $Y$ corresponding to $\Sigma$. Since a special case of this situation is when $\Gamma$ is cut out on $Y$ by the hyperplane spanned by $Q_{1}, \ldots, Q_{s}$, we see that the general $\Gamma$ is the general hyperplane section of $Y$. Since it imposes $2 s-1$ conditions on the quadrics, theorem 1.1 tells us that $\delta_{k}=1$.

Notice that the contact variety is irreducible in these cases.

Example 4.6. From the previous example we have that the 2-Veronese image $V_{2, s}$ of $\mathbf{P}^{s}$ in $\mathbf{P}^{r}$, with $r=\frac{s(s+3)}{2}$, is defective. Arguing as in the previous example, one sees that if $\frac{s}{2} \leq k \leq s-1$, then $\delta_{k}=\left(\begin{array}{c}s-k+1 \\ 2\end{array}\right)$. On the other hand, it is an exercise to see that for $1 \leq k \leq \frac{s}{2}$ one has $\delta_{k}=\left(\begin{array}{c}k+1 \\ 2\end{array}\right)$.

Let us now take any reduced, irreducible, non-degenerate, projective variety $X \subset V_{2, s}$ of dimension $n<s$. We make the following claims:

Claim 1: If $k$ is such that $1 \leq k \leq s-1$ and

$$
2(k+1)(n+1) \geq(s+2)(s+1),
$$

then $X$ is $k$-defective.

Claim 2: If $k$ is such that $1 \leq k \leq s-1$ and

$$
2(k+1)(n+1) \leq(s+2)(s+1)-2,
$$

and, in addition, $2(s-n)<k$, then again $X$ is $k$-defective.

Claim 3: If $k$ is such that $1 \leq k \leq s-1, k=2(s-n)$ and

$$
2(k+1)(n+1) \leq(s+2)(s+1)-2,
$$

then either $X$ is $k$-defective or it is $k$-weakly defective with $\nu_{k}=s-n$.

As for the proofs, notice that, under the hypothesis of claim 1, we have $\sigma^{(k)}(X)=$ $r$, and therefore we would expect $\mathcal{H}\left(-2 P_{1}-\cdots-2 P_{k+1}\right)$ to be empty for general points $P_{1}, \ldots, P_{k+1}$ of $X$. Instead, this system is not empty of dimension $\left(\begin{array}{c}s-k+1 \\ 2\end{array}\right)-$ 1 , since it coincides with the linear system of quadric cones in $\mathbf{P}^{s}$ with $k+1$ given independent double points. This proves claim 1.

For claim 2, the situation is similar. We have $r \geq(k+1)(n+1)$; hence

$$
\begin{aligned}
& \delta_{k}(X)=\operatorname{dim}\left(\mathcal{H}\left(-2 P_{1}-\cdots-2 P_{k+1}\right)\right)-r+(k+1)(n+1) \\
& \geq\left(\begin{array}{c}
s-k+1 \\
2
\end{array}\right)-\left(\begin{array}{c}
s+2 \\
2
\end{array}\right)+(k+1)(n+1)=\left(\begin{array}{c}
k+1 \\
2
\end{array}\right)-(k+1)(s-n)>0,
\end{aligned}
$$

which proves the claim.

As for claim 3, the above computation shows that, if $\delta_{k}(X)=0$, then the linear system $\mathcal{H}\left(-2 P_{1}-\cdots-2 P_{k+1}\right)$ has exactly dimension $\left(\begin{array}{c}s-k+1 \\ 2\end{array}\right)-1$. This implies that $\mathcal{H}\left(-2 P_{1}-\cdots-2 P_{k+1}\right)$ comes, via the 2 -Veronese map of $\mathbf{P}^{s}$, from the linear system cut out on $X$ by the quadric cones with vertex $\left\langle P_{1}, \ldots, P_{k+1}\right\rangle$. Notice that, 
abusing notation, we are identifying $X$ with its pre-image under the Veronese map. Since $\left\langle P_{1}, \ldots, P_{k+1}\right\rangle$ cuts $X$ in a subvariety of dimension $k+n-s=s-n$, claim 3 follows.

Notice that claim 3 yields that the 2-Veronese image of any surface of degree $d \geq 3$ in $\mathbf{P}^{3}$ is 2 -weakly defective.

Example 4.7. This is a variation and an extension of example 4.5. On the same lines example 4.5 could be further generalized, but we will not do so here.

Let us consider an irreducible, reduced, non-degenerate, projective variety $Y \subset$ $\mathbf{P}^{s}$ of dimension $n$ such that its general curve section $C$ is a linearly normal curve of arithmetic genus $g \geq 0$ and degree $d \geq 2 g+1$. One knows that $C$, and therefore $Y$, is then projectively Cohen-Macaulay and $d=s-n+g+1$ (see theorem 6.1 below). Note that the case $g=0$ gives us back the varieties of minimal degree considered in example 4.5 .

The quadrics in $\mathbf{P}^{s}$ cut out on $Y$ a complete linear system, which is of dimension

$$
r=s(n+1)-\frac{(n-2)(n+1)}{2}+g-1
$$

(see theorem 6.1, below). Then the 2-Veronese embedding $X$ of $Y$ in $\mathbf{P}^{r}$ is $k$ defective for every $k$ such that

$$
s-\left[\frac{n-2}{2}\right]-1 \leq k \leq s-1
$$

as soon as $\left(\begin{array}{c}s-k+1 \\ 2\end{array}\right)>g$. Indeed, one has

$$
r-(n+1)(k+1) \leq r-(n+1)\left(s-\left[\frac{n-2}{2}\right]\right) \leq r-(n+1)\left(s-\frac{n-2}{2}\right)=g-1,
$$

whereas, with the same argument we used in example 4.5 , we can see that $\mathcal{H}\left(-2 P_{1}-\cdots-2 P_{k+1}\right)$ is not empty, of dimension at least $\left(\begin{array}{c}s-k+1 \\ 2\end{array}\right)-1$.

The situation is particularly interesting if $g=1$. Then the above argument shows that $X$ is $k$-defective for some $k$, unless $n=2$. In this case, however, the surface $S$ is $(s-1)$-weakly defective but not $(s-1)$-defective. In fact we have $\sigma^{(s-1)}=r-1$; hence we expect $\mathcal{H}\left(-2 P_{1}-\cdots-2 P_{s}\right)$ to be 0 -dimensional. Notice that $\mathcal{H}\left(-2 P_{1}-\cdots-2 P_{s}\right)$ contains the double of the hyperplane section $\Gamma$ of $Y$ passing through the points $Q_{1}, \ldots, Q_{s}$ corresponding to $P_{1}, \ldots, P_{s}$. Observe that $\Gamma$ is a general hyperplane section of $Y$ and $Q_{1}, \ldots, Q_{s}$ are general points on it. Suppose there is a divisor $D$ in $\mathcal{H}\left(-2 P_{1}-\cdots-2 P_{s}\right)$ not containing $\Gamma$. Then it should cut out on $\Gamma$ the divisor $2 Q_{1}+\cdots+2 Q_{s}$, which therefore would belong to $\left|\mathcal{O}_{\Gamma}(2)\right|$, contrary to the generality assumption on $Q_{1}, \ldots, Q_{s}$. Thus $D$ contains $\Gamma$, i.e. $D=\Gamma+\Gamma^{\prime}$, with $\Gamma^{\prime}$ linearly equivalent to $\Gamma$, containing $Q_{1}, \ldots, Q_{s}$. Again by the generality assumption on $Q_{1}, \ldots, Q_{s}$, one has $\Gamma=\Gamma^{\prime}$, proving that $\mathcal{H}\left(-2 P_{1}-\cdots-2 P_{2}\right)$ is indeed 0 -dimensional, and therefore $X$ is not $(s-1)$-defective. On the other hand, since the unique element in $\mathcal{H}\left(-2 P_{1}-\cdots-2 P_{s}\right)$ is $2 \Gamma$, we see that $X$ is $(s-1)$-weakly defective, and the contact curve is irreducible.

Notice that in the above situation of surfaces $Y \subset \mathbf{P}^{s}$, of degree $s$ with curve sections of arithmetic genus 1 , one has to take, in principle, $s \geq 3$ in order to make a sensible construction. However, one can also consider double covers $f: Y \rightarrow \mathbf{P}^{2}$ branched along a quartic curve. Then the linear system $\left|f^{*}(L)\right|$, where $L$ is a line in $\mathbf{P}^{2}$, consists of elliptic curves, and $\left|2 f^{*}(L)\right|$ determines an embedding of $Y$ in $\mathbf{P}^{6}$ as a surface $X$ which is 1 -weakly defective, as one can see with the same argument 
as above. However $X$ also sits on the cone over the Veronese surface in $\mathbf{P}^{5}$; thus these examples are also included in example 4.4.

As for the classification of surfaces $Y \subset \mathbf{P}^{s}$, of degree $s$ with curve setions of arithmetic genus 1, it is very well known that, if the general hyperplane section of $Y$ is smooth, then either $s \leq 9$ and $Y$ is a del Pezzo surface or $Y$ is a cone (for a quick proof of this classical result, see CiLoMi]). If the general hyperplane section of $Y$ is singular, then it has either a node or a cusp, hence $Y$ itself has either a nodal or a cuspidal line, and it is a projection of a rational normal scroll $Y^{\prime} \subset \mathbf{P}^{s+1}$ of degree $s$, from a point $P \notin Y^{\prime}$ lying in a plane which cuts $Y^{\prime}$ along a conic, whose projection in $\mathbf{P}^{s}$ is the double line.

\section{Involutions on varieties and Castelnuovo-Humbert's theorem}

The present section contains a basic preliminary, of independent interest, to the proof of the classification theorem 1.3. Namely, we prove an extension to higher dimensional varities of a famous theorem of Castelnuovo and Humbert on involutions on curves (see [EnChi], pg. 480; [Ma, Appendix).

Let $X$ be a reduced, irreducible, projective variety of dimension $n$. Let $\mathcal{D}=$ $\left\{D_{y}\right\}_{y \in Y}$ be an algebraic family of Weil divisors on $X$ parametrized by a reduced variety $Y$. We will constantly assume in what follows that $\mathcal{D}$ is effectively parametrized by $Y$, i.e. the corresponding map of $Y$ to the appropriate Hilbert scheme of divisors on $X$ is generically finite to the image on any irreducible component of $Y$. We will say that $\mathcal{D}$ is irreducible of dimension $m$ if $Y$ is, and we can speak in an obvious way of the irreducible components of $Y$, etc.

Let $P_{1}, \ldots, P_{h}$ be points in $X$. We define $\mathcal{D}\left(-P_{1}-\cdots-P_{h}\right)$ to be the family of all divisors in $\mathcal{D}$ containing $P_{1}, \ldots, P_{h}$.

Suppose $\mathcal{D}$ has pure dimension $m$. For every positive integer $h$ we can consider the incidence correspondence

$$
I^{(h)}:=I^{(h)}(\mathcal{D})=\left\{\left(y, P_{1}, \ldots, P_{h}\right) \in Y \times X^{h}: P_{1}, \ldots, P_{h} \in D_{y}\right\}
$$

with the two projections $p_{1}: I^{(h)} \rightarrow Y$ and $p_{2}: I^{(h)} \rightarrow X^{h}$. All the fibres of $p_{1}$ have the same dimension $h(n-1)$; hence all the irreducible components of $I^{(h)}$ have the same dimension $(h+1)(m-1)+1$ and dominate $Y$ via $p_{1}$.

Lemma 5.1. Suppose $\mathcal{D}$ is irreducible of positive dimension $m$.

(i) For every positive integer $h$ there is at least one irreducible component $J$ of $I^{(h)}$ such that the map $p_{2}: J \rightarrow X^{h}$ is generically of maximal rank; namely, it is dominant if and only if $h \leq m$, it is generically finite to the image if and only if $h \geq m$, and both generically finite and dominant if and only if $h=m$.

(ii) Let $h$ be a positive integer such that $h \leq m$. If $P_{1}, \ldots, P_{h}$ are general points, then every irreducible component of $\mathcal{D}\left(-P_{1}-\cdots-P_{h}\right)$ has dimension $m-h$. If $P_{1}, \ldots, P_{h}$ are points in $X$, then every irreducible component of $\mathcal{D}\left(-P_{1}-\cdots-P_{h}\right)$ has dimension at least $m-h$.

Proof. First we prove (i) for $h \leq m$. In this case it suffices to prove that $p_{2}: I^{(h)} \rightarrow$ $X^{h}$ is dominant.

The assertion is true for $h=1$, since $\mathcal{D}$ is effectively parametrized by a positive dimensional variety $Y$, and therefore $D_{y}$ moves as $y$ moves in $Y$, thus describing a Zariski dense subset of $X$. So we may argue by induction on $h$.

Consider the projection $\pi: X^{k} \rightarrow X$ to the last factor and the composite maps $f=\pi \circ p_{2}: I^{(h)} \rightarrow X^{k-1}$ and $f_{2}=\pi_{2} \circ p_{2}: I^{(h)} \rightarrow X$, which are again 
dominant. Hence its fibre $\Phi$ over a general point $P_{h}$ is pure of dimension equal to $\operatorname{dim}\left(I^{(h)}\right)-\operatorname{dim}(X)=n h+m-h-n$. We may replace $\Phi$ by one of its components and assume it is irreducible. The general fibre $\Psi$ of $p_{1}: \Phi \rightarrow Y$ has dimension at most $(h-1)(n-1)$; hence $Y^{\prime}:=p_{1}(\Phi)$ is irreducible of dimension at least $m-1$. Since $Y^{\prime}$ cannot coincide with $Y$, because all divisors $D_{y}$ with $y \in Y^{\prime}$ contain $P_{h}$ which is a general point on $X$, we have that $Y^{\prime}$ has codimension 1 in $Y$. Consider the family $\mathcal{D}^{\prime}=\left\{D_{y}\right\}_{y \in Y^{\prime}}$. It is clear that, as $P_{h}$ moves in $X, Y^{\prime}$ moves with $P_{h}$ sweeping out the whole of $Y$. This implies that $\mathcal{D}^{\prime}$ is effectively parametrized by $Y^{\prime}$. So, by applying induction, one easily concludes the proof of (i) in this case.

Now let us prove the first assertion of (ii). Let $J$ be any irreducible component of $I^{(h)}$ and let $F_{J}$ be the fibre of $p_{2}: J \rightarrow X^{h}$ over a general point $\left(P_{1}, \ldots, P_{h}\right)$ in $X^{h}$. Then either $p_{2}: J \rightarrow X^{h}$ is dominant, in which case $F_{J}$ has pure dimension $m-h=\operatorname{dim}(J)-\operatorname{dim}\left(X^{h}\right)$, or $F_{J}$ is empty. The first possibility actually occurs for some $J$, as we already proved.

Notice that $p_{1}$ is injective on $F_{J}$ and $\mathcal{D}\left(-P_{1}-\cdots-P_{h}\right)$ is the union of the $F_{J}$ 's as $J$ varies among the irreducible components of $I$; hence it is not empty, of dimension $m-h$.

The proof of the second assertion of (ii) is similar, and therefore we omit it.

Finally we prove (i) for $h>m$. The projection $\pi_{h, m}: X^{h} \rightarrow X^{m}$ onto the first $m$ factors induces a surjective map $f_{h, m}: I^{(h)} \rightarrow I^{(m)}$ whose fibres all have dimension $(h-m)(n-1)$. Thus $f_{h, m}$ carries irreducible components $J$ of $I^{(h)}$ onto irreducible components $J^{\prime}$ of $I^{(m)}$. Let $J^{\prime}$ be an irreducible component of $I^{(m)}$ which dominates $X^{m}$. Such a component exists, as we already proved. Let $J$ be a component of $I^{(h)}$ dominating $J^{\prime}$ via $f_{h, m}$ and let $\left(y, P_{1}, \ldots, P_{h}\right)$ be a general element of $J$. Then $\left(y, P_{1}, \ldots, P_{m}\right)$ is a general element of $J^{\prime}$, and so $P_{1}, \ldots, P_{m}$ are general points on $X$. Since (ii) holds for $h=m$, then $\mathcal{D}\left(-P_{1}-\cdots-P_{m}\right)$ is finite, which implies that also $\mathcal{D}\left(-P_{1}-\cdots-P_{h}\right)$ is finite. This implies that the map $p_{2}: J \rightarrow X^{h}$ is generically finite to the image, proving (i) in this case.

Let $X$ be as above and let $\mathcal{D}$ be a family of pure positive dimension $m$ of divisors on $X$. If $P_{1}, \ldots, P_{m}$ are general points of $X$, then we know by lemma 5.1 , ii, that $\mathcal{D}\left(-P_{1}-\cdots-P_{m}\right)$ is finite. The number of its elements is called the index of $\mathcal{D}$. We will say that $\mathcal{D}$ is an involution if its index is 1 .

Lemma 5.2. Suppose $\mathcal{D}$ is an involution on the irreducible, reduced variety $X$. Then:

(i) there is a unique irreducible component $J$ of $I^{(m)}$ dominating $X^{m}$ via $p_{2}$;

(ii) $p_{2}: J \rightarrow X^{m}$ is a birational morphism;

(iii) $\mathcal{D}$ is irreducible; and

(iv) if $\mathcal{D}$ has no fixed divisor, then $I^{(1)}$ is irreducible.

Proof. Assertions (i) and (ii) are immediate consequences of the definition. By lemma 5.1, i, assertion (iii) follows from (i).

Let us prove (iv). Since $\mathcal{D}$ has no fixed divisor, every irreducible component of $I^{(1)}$ dominates $X$ via $p_{2}$. Now $I^{(m)}$ is the $m$-fold fibred product of $I^{(1)}$ over $Y$. Thus two different irreducible components of $I^{(1)}$ would give rise to two different irreducible components of $I^{(m)}$, both dominating $X^{m}$ via $p_{2}$, against (i).

The next examples show typical situations in which we have involutions on a variety: 
Example 5.3. A linear system is clearly an involution.

Example 5.4. If $X$ is a curve, the family $\mathcal{D}_{m}:=\mathcal{D}_{m}(X)$ of all divisors of degree $m$ on $X$ is an $m$-dimensional involution.

Example 5.5. Let $X$ be a variety as above and let $V$ be an irreducible, reduced projective variety. Let $f: X \rightarrow V$ be a rational dominant map which is not the identity and let $\mathcal{D}$ be an algebraic family on $V$. The pull-back $f^{*}(\mathcal{D})$ of $\mathcal{D}$ on $X$ is defined in the obvious way. Then $\mathcal{D}$ is an involution if and only if the same is true for $f^{*}(\mathcal{D})$, which is said to be composite with the map $f$.

In particular, if $V$ is a curve, then the family $\mathcal{D}_{f, m}=f^{*}\left(\mathcal{D}_{m}(V)\right)$ whose general member is a sum of $m$ general fibres of $f$ is an $m$-dimensional involution on $X$. We write $\mathcal{D}_{f}$ instead of $\mathcal{D}_{f, 1}$. If an involution $\mathcal{D}$ is composed with such an $f$, we also say that it is composite with the invoution $\mathcal{D}_{f}$. The involution $\mathcal{D}_{f}$, or the map $f$, is said to be a pencil if $f$ has irreducible fibres. Using Stein factorization, one sees that every involution of the type $\mathcal{D}_{f}$ is compososite with a pencil.

Recall that the theorem of Bertini asserts that if $\mathcal{D}$ is a linear system without a fixed part, whose general member is reducible, then it is composed with a pencil.

Example 5.6. Let $\mathcal{D}$ be a family of divisors on $X$. If we count any divisor in $\mathcal{D}$ with a certain multiplicity $\mu \geq 2$, we have a new family $\mathcal{D}^{(\mu)}$ which is an involution if and only if $\mathcal{D}$ is.

Next we give a criterion which tells us when an algebraic family is composed with a pencil.

Proposition 5.7. Let $\mathcal{D}$ be an irreducible family of divisors on a reduced, irreducible variety $X$ of dimension $n \geq 2$ [resp. of dimension $n=1$ ]. If $\mathcal{D}$ has no fixed divisor, then it is composed with a pencil if and only if for a general point $P$ in $X$, the family $\mathcal{D}(-P)$ has a fixed divisor [resp. a fixed divisor of degree at least 2].

Proof. We only prove the non-trivial implication. We also assume $n \geq 2$, the curve case being similar. Suppose $\mathcal{D}(-P)$ has a fixed divisor $\Phi$. Let $F$ be an irreducible component of $\Phi$. Since for $Q \in F$ general we have $\mathcal{D}(-P)=\mathcal{D}(-Q)$ by 5.1 , ii, then $P$ sits, by its generality, as a general point on some irreducible component $F_{P}$ of $\Phi$. As $P$ varies on $X, F_{P}$ varies in some component $W$ of the Hilbert scheme of divisors on $X$. Hence we have a map $f: P \in X \rightarrow\left[F_{P}\right] \in W$, whose general fibre is exactly $F_{P}$. Hence $C:=f(X)$ is a curve and $f: X \rightarrow C$ is a pencil. Furthermore, if $D$ is a general divisor in $\mathcal{D}$, then $D$ is the sum of divisors in the pencil $\mathcal{D}_{f}$. This follows by the obvious fact that for any general point $P$ in a component $D^{\prime}$ of $D$, and $F_{P}$ is contained in $D$; hence coincides with $D^{\prime}$. This description of $\mathcal{D}$ immediately yields the assertion.

We also point out the following proposition:

Proposition 5.8. Let $X$ be a reduced, irreducible variety. Let $\mathcal{D}$ be an involution on $X$ which has no fixed component. If the general divisor $D$ in $\mathcal{D}$ is non-reduced, then there is an involution $\mathcal{E}$ whose general divisor is reduced and there is an integer $\mu \geq 2$ such that $\mathcal{D}=\mathcal{E}^{(\mu)}$.

Proof. We know by lemma 5.2, iv, that $I^{(1)}$ is irreducible. Let us consider the normalization $\pi: I \rightarrow I^{(1)}$ with the two maps $\pi_{1}:=\pi \circ p_{1}: I \rightarrow Y$ and $\pi_{2}:=\pi \circ p_{2}$ : $I \rightarrow X$. The general fibre of $\pi_{1}$ is smooth. Let us consider the Stein factorization of $\pi_{1}$. We have an $m$-dimensional variety $T$, a finite morphism $\psi: T \rightarrow Y$ of a 
certain degree $\delta$, and a morphism $\phi: I \rightarrow T$ with smooth and irreducible general fibres, such that $\pi_{1}: \phi \circ \psi$. Now $\phi: I \rightarrow T$ may be interpreted as a new family $\mathcal{D}^{\prime}=\left\{F_{t}\right\}_{t \in T}$ of divisors on $X$. The divisors of $\mathcal{D}$ are sums of $\delta$ divisors of $\mathcal{D}^{\prime}$. More precisely If $y \in Y$ is a general point and $t_{1}, \ldots, t_{\delta}$ are the points in $\psi^{-1}(y)$, then $D_{y}=\mu_{1} F_{t_{1}}+\cdots+\mu_{\delta} F_{t_{\delta}}$, where $\mu_{1}, \ldots, \mu_{\delta}$ are integers. However, since $T$ is irreducible, the monodromy of $\psi: T \rightarrow Y$ acts in an irreducible way on $\psi^{-1}(y)$, yielding $\mu_{1}=\cdots=\mu_{\delta}$. This immediately implies our assertion.

The classical Castelnuovo-Humbert theorem for curves can be stated as follows:

Proposition 5.9. Let $X$ be a reduced, irreducible curve and let $\mathcal{D}$ be an m-dimensional involution on $X$ which has no fixed component, whose general divisor is reduced, and which is not composed with a map $f: X \rightarrow C$ of degree $d \geq 2$ to a curve $C$ of positive genus. Then either $\mathcal{D}$ is a linear system or $\mathcal{D}=\mathcal{D}_{m}$.

Proof. Let $m=1$, i.e. the parameter variety $Y$ of $\mathcal{D}$ is a curve $C$. Then $I^{(1)}$ is an irreducible curve which is birational to $X$ via $p_{2}$. Consider the rational dominant map $f: p_{1} \circ p_{2}^{-1}: X \rightarrow C$. Clearly $\mathcal{D}=\mathcal{D}_{f}$. Hence our assumptions imply that either $f$ has degree 1 , i.e. $\mathcal{D}=\mathcal{D}_{1}$, or $C$ is rational, i.e. $\mathcal{D}$ is a linear system.

Now let $m \geq 2$. Let us denote by $d$ the degree of the divisors of $\mathcal{D}$. We have $d \geq m$, and equality holds if and only if $\mathcal{D}=\mathcal{D}_{m}$. Hence we may assume $d \geq 3$. We argue by induction. Since the problem is birational in nature, we may, and will, assume $X$ is smooth.

Let $P$ be a general point of $X$ and consider $\mathcal{D}(-P)$. By the assumptions and by proposition 5.7, $P$ is the only fixed part of $\mathcal{D}(-P)$. Let $\mathcal{D}_{P}$ be the fixed point free involution obtained from $\mathcal{D}(-P)$ by taking $P$ away from the general divisor of $\mathcal{D}$ containing it.

Claim: $\mathcal{D}_{P}$ is not composite with a map $f: X \rightarrow C$ of degree $d \geq 2$ onto a curve $C$ of positive genus.

Let us argue by contradiction. By the results in the appendix of $\mathrm{Ma}$, we see that $f$ would not depend on $P$. Take a general divisor $D \in \mathcal{D}_{P}$. Let $Q$ be a point in $D$ different from $P$. $Q$ is another general point of $X$. Thus $D-Q \in \mathcal{D}_{Q}$. Then $D-Q$ should contain $f^{*}(f(P))$, and $\mathcal{D}$ itself would be composite, by (5.7), with $f$, a contradiction.

By the claim, we may apply induction to $\mathcal{D}_{P}$. Then either $\mathcal{D}_{P}=\mathcal{D}_{m-1}$, which yields $\mathcal{D}=\mathcal{D}_{m} ;$ or $\mathcal{D}_{P}$ is a linear system. In the latter case we see that if $D \in \mathcal{D}$ is a general divisor, there are $d$ linear systems of dimension $m-1$ of the type $\mathcal{D}_{P}$, with $P \in D$, containing $D$. Since each of these systems is mapped to a point via the natural map $Y \rightarrow \operatorname{Pic}(X)$, we see that $Y$ is mapped to a point, i.e. $\mathcal{D}$ is contained in a complete linear system $\mathcal{L}$ of dimension $r \geq m$. Then we have the natural map $y \in Y \rightarrow D_{Y} \in \mathcal{L}=\mathbf{P}^{r}$ that is generically finite to the image $Y^{\prime}$, which therefore has dimension $m$. The above argument shows that, given a general point of $Y^{\prime}$, we have at least $d \geq 3$ subspaces $\mathbf{P}^{m-1}$ passing through that point and contained in $Y^{\prime}$. Hence $Y^{\prime}$ is ruled with at least $d \geq 3$ rulings. This implies that $Y^{\prime}$ is a projective space. This is clear if $m=2$ : a ruled surfaces which is not $\mathbf{P}^{2}$ has at most two different rulings, in which case it is a quadric. If $m>2$, one reduces to the surface case by considering a general surface section. This concludes our proof. 
Our extension of the Castelnuovo-Humbert theorem to higher dimensional varieties reduces all involutions either to linear systems or to involutions on curves already classified in theorem 5.9 .

Theorem 5.10. Let $X$ be a reduced, irreducible variety of dimension $n>1$. Let $\mathcal{D}$ be an $m$-dimensional involution on $X$ which has no fixed divisor and whose general divisor $D$ is reduced. Then either $\mathcal{D}$ is a linear system, or it is composite with a pencil.

Proof. Since the problem is birational, we may, and will, assume $X$ is smooth. We will freely use here the construction and notation from the proof of proposition 5.6.

Suppose first that the general divisor $D \in \mathcal{D}$ is irreducible. In the present case one has $T=Y$.

Let $m=1$, i.e. $Y$ is a curve $C$. The map $\pi_{2}: I \rightarrow X$ is now birational. Hence the rational map $f:=\pi_{1} \circ \pi_{2}^{-1}: X \rightarrow C$ is dominant, and of course $\mathcal{D}=\mathcal{D}_{f}$.

Suppose next that $m \geq 2$. We will argue by induction. Let $P$ be a general point of $X$. Then $\mathcal{D}(-P)$ is an $(m-1)$-dimensional involution. Since in the present case clearly $\mathcal{D}$ is not composite with a pencil, by proposition 5.7 the system $\mathcal{D}(-P)$ has no fixed divisor. Then, by induction, $\mathcal{D}(-P)$ is a linear system of dimension $m-1 \geq 1$. If $D \in \mathcal{D}$ is a general divisor and $P$ is a general point on $D$, then $\mathcal{D}(-P)$ is a linear system contained in $\mathcal{D}$ and containing $D$. Furthermore, $\mathcal{D}(-P)$ is different from $\mathcal{D}(-Q)$, if $P$ and $Q$ are two general points of $D$. This immediately implies that the natural map $Y \rightarrow \operatorname{Pic}(X)$ is constant, i.e. $\mathcal{D}$ is contained in a linear system. We want to prove now that $\mathcal{D}$ itself is a linear system.

Take general divisors $D, D^{\prime} \in \mathcal{D}$. Let $Z$ be the scheme-theoretic intersection of $D$ and $D^{\prime}$. First we notice that $Z \neq \emptyset$. Otherwise we would have $\operatorname{dim}|D| \leq 1$, contrary to the fact that $\operatorname{dim}|D| \geq \operatorname{dim} \mathcal{D}=m \geq 2$. Furthermore we claim that, as $D^{\prime}$ varies in $\mathcal{D}, Z$ describes a dense Zariski subset of $D$. Otherwise, since $D$ is irreducible, $Z$ would stay fixed. By blowing up $Z$ we would then reduce to the case $Z=\emptyset$, which we have already excluded. This implies that we can choose a general point $P$ on $X$ in such a way that it lies on $D \cap D^{\prime}$. Hence $D$ and $D^{\prime}$ are connected by the $(m-1)$-dimensional linear system $\mathcal{D}(-P)$ inside $\mathcal{D}$. This proves that $\mathcal{D}$ itself is a linear system.

Suppose now that the general divisor $D \in \mathcal{D}$ is reducible. We will prove that $\mathcal{D}$ is composite with a pencil in this case. The case $m=1$ can be easily treated as before. Let $m \geq 2$, and let us argue by induction.

Let $P$ be a general point on $X$. Suppose $\mathcal{D}(-P)$ has no fixed divisor. By induction there are a pencil $f: X \rightarrow C$ and an involution $\mathcal{E}$ on $C$ such that $\mathcal{D}(-P)=f^{*} \mathcal{E}$. Since all divisors in $\mathcal{D}(-P)$ contain $P$, then all fibres of $f$ contain $P$. Let $d>1$ be the degree of divisors in $\mathcal{E}$. Then a general divisor $D$ in $\mathcal{D}(-P)$ would consist of $d$ fibres of $f$, all passing through $P$. Hence $D$ would be singular at $P$, against the generality of $P$ and $D$. In conclusion, $\mathcal{D}(-P)$ has a fixed divisor, and we are done by proposition 5.7 .

Remark 5.11. This remark has little to do with the main object of the present section, i.e. the Castelnuovo-Humbert theorem. However, it has to do with families of divisors, and it will be useful later on. Let $X$ be a reduced, irreducible variety of dimension $n$ and let, as usual, $\mathcal{D}=\left\{D_{y}\right\}-y \in Y$ be an algebraic family of Weil divisors on $X$ parametrized by a reduced variety $Y$ of dimension 1 . If $P$ is a general 
point of $X$, then any two divisors in $\mathcal{D}(-P)$ intersect transversally at $P$. The proof relies on a straightforward local computation, and it is left to the reader.

\section{A Basic Result from CASTElnuovo's theory}

The present section is devoted to a basic result in Castelnuovo's theory which is a slight extension of propostion 3.23 from [EiHa]. We used part of this result already in examples 4.5 and 4.7, and we will rely on it in our proof of theorem 1.3.

Before stating the theorem, we introduce some notation and definitions. Let $X$ be a scheme in $\mathbf{P}^{r}$ of positive pure dimension $n$ and codimension $q=r-n$. We denote by $h_{X}$ the Hilbert function of $X$, i.e., for any integer $l$ we set

$$
h_{X}(l)=\operatorname{dim}\left(\operatorname{Im}\left(\rho_{X, l}: H^{0}\left(\mathbf{P}^{r}, \mathcal{O}_{\mathbf{P}^{r}}(l)\right) \rightarrow H^{0}\left(X, \mathcal{O}_{X}(l)\right)\right) .\right.
$$

We will also use the first difference function $\Delta h_{X}(l):=h_{X}(l)-h_{X}(l-1)$.

We recall that $X$ is said to be l-normal if the map $\rho_{l}$ is surjective, which is equivalent to saying that $H^{1}\left(\mathbf{P}^{r}, \mathcal{I}_{X}(l)\right)=0$, where $\mathcal{I}_{X}$ is the ideal sheaf of $X$ in $\mathbf{P}^{r}$. One says that $X$ is linearly [resp. quadratically, etc.] normal, if it is 1-normal [resp. 2-normal, etc.].

If $X$ is $l$-normal for all $l \geq 0$, then one says that $X$ is arithmetically normal. Let $X_{i}$ be the section of $X$ with a general $\mathbf{P}^{q+i}, i=0, \ldots, n$. Notice that $X_{n}=X$.

If $X_{i}$ is arithmetically normal for all $i=1, \ldots, n$, one has that $X$ is projectivelly Cohen-Macaulay, i.e. the coordinate ring of $X$ in $\mathbf{P}^{r}$ ia a Cohen-Macaulay ring.

Now we need to recall two basic facts from Castelnuovo's theory. The first one is that

$$
\Delta h_{X}(l)-h_{X_{n-1}}(l)=\operatorname{dim}\left(\operatorname{Ker}\left(\sigma_{X, l}: H^{1}\left(\mathbf{P}^{r}, \mathcal{I}_{X}(l-1)\right) \rightarrow H^{1}\left(\mathbf{P}^{r}, \mathcal{I}_{X}(l)\right)\right)\right) \geq 0,
$$

and the equality holds for all integers $l$ if and only if $X$ is projectively CohenMacaulay (see [Ci], §1).

The second basic result is that, if $X$ is irreducible of degree $d$, then

$$
h_{X_{0}}(l) \geq \min \{d, l q+1\}
$$

(see [EiHa], chapt. 3, or again [Ci], §1).

We are now ready to prove the following theorem.

Theorem 6.1. Let $X$ be a reduced, irreducible, non-degenerate, projective variety in $\mathbf{P}^{r}$ of positive dimension $n$ and codimension $q=r-n$ and of degree $d$. Suppose that $d \geq q+1+\iota$, with $0 \leq \iota \leq c$. Then for every integer $l \geq 2$ one has

$$
h_{X}(l) \geq\left(\begin{array}{c}
l+n-2 \\
n
\end{array}\right) \iota+\left(\begin{array}{c}
l+n-1 \\
n
\end{array}\right) q+\left(\begin{array}{c}
l+n \\
n
\end{array}\right) .
$$

If $d \leq 2 q+1$, then the following propositions are equivalent:

(i) equality holds in (6.3) for some $l \geq 2$;

(ii) equality holds in (6.3) for all $l \geq 2$;

(iii) the general curve section $X_{1}$ of $X$ is linearly normal of degree $d=q+1+\iota$;

(iv) $X$ is projectively Cohen-Macaulay of degree $d=q+1+\iota$.

Proof. By (6.1) we have

$$
h_{X}(l) \geq \sum_{i=1}^{n} h_{X_{i}}(l-1)+h_{X_{0}}(l)
$$


Since by the non-degeneracy hypothesis we have $h_{X_{i}}(1)=q+i+1, i=1, \ldots, n$, then (6.4) and (6.2) imply (6.3) for $l=2$. For $l \geq 3$ one proves (6.3) by induction.

Suppose equality holds in (6.3) for some $l \geq 2$. Then it has to hold for $l=2$. This implies that $d=q+1+\iota$ and that the map $\sigma_{X_{i}, 2}$ is injective for all $i=1, \ldots, n$.

Consider now, for every integer $l$ and for every $i=1, \ldots, n$, the exact sequence

$$
\cdots \rightarrow H^{1}\left(\mathbf{P}^{q+i}, \mathcal{I}_{X_{i}}(l-1)\right) \rightarrow H^{1}\left(\mathbf{P}^{q+i}, \mathcal{I}_{X_{i}}(l)\right) \rightarrow H^{1}\left(\mathbf{P}^{q+i-1}, \mathcal{I}_{X_{i-1}}(l)\right) \rightarrow \cdots .
$$

The injectivity of $\sigma_{X_{i}, 2}$ implies that $H^{1}\left(\mathbf{P}^{q+i}, \mathcal{I}_{X_{i}}(l)\right)=0$ for $l=1,2$, whenever the cohomology group $H^{1}\left(\mathbf{P}^{q+i-1}, \mathcal{I}_{X_{i-1}}(1)\right)$ vanishes. Since (6.2) implies that $H^{1}\left(\mathbf{P}^{q}, \mathcal{I}_{X_{0}}(l)\right)=0$ for all $l \geq 2$, we conclude, by induction, that $H^{1}\left(\mathbf{P}^{q+i}, \mathcal{I}_{X_{i}}(l)\right)=$ 0 for $l=1,2$, and all $i=1, \ldots, n$. Finally, by using (6.5) and induction again we conclude that $H^{1}\left(\mathbf{P}^{q+i}, \mathcal{I}_{X_{i}}(l)\right)=0$ for all $i=1, \ldots, n$ and for all integers $l$. We have thus proved that (i) implies (iv).

If (iv) holds, the proof of (6.3) shows that the equality has to hold there for all integres $l$. Thus (iv) implies (ii). Since (ii) implies (i), we see that (i), (ii) and (iv) are equivalent.

Of course (iv) implies (iii). Suppose (iii) holds. Notice that the irreducibility of $X$ implies that $X_{i}$ is irreducible for all $i=1, \ldots, n$. Hence $H^{1}\left(\mathbf{P}^{q+i}, \mathcal{I}_{X_{i}}\right)=0$ for all $i=1, \ldots, n$. Since (iii) implies that $H^{1}\left(\mathbf{P}^{q+1}, \mathcal{I}_{X_{1}}(1)\right)=0$, we see by induction, using (6.5) for $l=1$, that $H^{1}\left(\mathbf{P}^{q+i}, \mathcal{I}_{X_{i}}(1)\right)=0$ for all $i=1, \ldots, n$. Then, proceeding as before by induction, we conclude again that $H^{1}\left(\mathbf{P}^{q+i}, \mathcal{I}_{X_{i}}(l)\right)=0$ for all $i=1, \ldots, n$ and for all integers $l$. We have thus proved that (iii) is equivalent to (iv).

\section{The PROOF OF THE CLASSIFICATION THEOREM}

In this section we can finally give the

Proof of the classification theorem (1.3). Let $X \subset \mathbf{P}^{r}$ be a reduced, irreducible, non-degenerate, projective, $k$-weakly defective surface. If $k=0$, we know by remark 3.1 , ii, that $X$ is either a cone or the tangent developable to a curve. Thus from now on we will assume $k \geq 1$.

Let $P_{1}, \ldots, P_{k+1}$ be general points on $X$. Let $H$ be a general hyperplane section in $\mathcal{H}\left(-2 P_{1}-\cdots-2 P_{k+1}\right)$. As in $\S 1$, we consider $\Sigma:=\Sigma_{P_{1}, \ldots, P_{k+1}}(H)$, which is the union of all irreducible components of $\operatorname{Sing}(H)$ containing $P_{1}, \ldots, P_{k+1}$.

Recalling theorem (1.1), since $\nu_{k}=1$ in this case, we have

$$
h(\Sigma)=r-\operatorname{dim}(\mathcal{H}(-\Sigma)) \leq 2(k+1)-\delta_{k} \leq 2(k+1) .
$$

Bertini's theorem implies

Claim 1: For a general $H$ in $\mathcal{H}\left(-2 P_{1}-\cdots-2 P_{k+1}\right)$, the set $\Sigma_{P_{1}, \ldots, P_{k+1}}(H)$ does not depend on $H$.

Furthermore, by the very defintion of $\Sigma_{P_{1}, \ldots, P_{k+1}}(H)$, one has

Claim 2: $\Sigma_{P_{1}, \ldots, P_{k+1}}(H)$ has no fixed components as $P_{1}, \ldots, P_{k+1}$ vary on $X$ and $H$ varies in $\mathcal{H}\left(-2 P_{1}-\cdots-2 P_{k+1}\right)$.

Indeed, each irreducible component of $\Sigma_{P_{1}, \ldots, P_{k+1}}(H)$ has to contain at least one of the points $P_{1}, \ldots, P_{k+1}$, each one of which varies describing the whole of $X$.

Proposition 2.4 yields 
Claim 3: If $P_{1}, \ldots, P_{k+1}$ are general points on $X$ and if $H$ is general in the linear system $\mathcal{H}\left(-2 P_{1}-\cdots-2 P_{k+1}\right)$, then every irreducible component of $\Sigma_{P_{1}, \ldots, P_{k+1}}(H)$ appears with multiplicity 2 in $H$.

Then we can write

$$
H=2 \Sigma_{P_{1}, \ldots, P_{k+1}}(H)+\Delta_{P_{1}, \ldots, P_{k+1}}(H)
$$

where $\Delta:=\Delta_{P_{1}, \ldots, P_{k+1}}(H)$ is effective. As $H$ varies in $\mathcal{H}\left(-2 P_{1}-\cdots-2 P_{k+1}\right), \Delta$ varies in a linear system $\mathcal{L}:=\mathcal{L}_{P_{1}, \ldots, P_{k+1}}$ of dimension.

$$
\operatorname{dim}\left(\mathcal{L}_{P_{1}, \ldots, P_{k+1}}\right)=r-3(k+1)+\delta_{k} .
$$

Thus

$$
r=3(k+1)-\delta_{k}+\operatorname{dim}\left(\mathcal{L}_{P_{1}, \ldots, P_{k+1}}\right) \geq 3(k+1)-\delta_{k} .
$$

The claims above imply that $\Sigma$ varies in a $(k+1)$-dimensional involution $\mathcal{D}$ on $X$, with no fixed component, whose general divisor is reduced. Now we discuss separately the two cases: (i) the general divisor $\Sigma$ is irreducible; (ii) the general divisor $\Sigma$ is reducible.

Case (i): The general divisor $\Sigma$ is irreducible.

By theorem $5.10, \mathcal{D}$ is a linear system of dimension $k+1 \geq 2$.

Claim 4: In formula (7.2) one has $\Delta:=\Delta_{P_{1}, \ldots, P_{k+1}}(H)=0$.

First we prove the assertion for $k=1$. Proposition 3.3, iv, tells us that $\delta_{1} \leq 1$. Then (7.3) yields $r \geq 6-\delta_{1} \geq 5$.

If $X$ is 1 -defective, then (7.1) implies that the curves $\Sigma$ are irreducible plane curves. They are non-degenerate plane curves, as otherwise $X$ would contain a 2-dimensional family of lines; hence it would be a plane, against its non-degeneracy in $\mathbf{P}^{r}$. But then it is a classical result that $X$ is the Veronese surface in $\mathbf{P}^{5}$ (for a modern reference see [CiSe], whence the assertion immediately follows.

Suppose now $X$ is not 1-defective. By (7.1) each of the curves $\Sigma$ lies in some $\mathbf{P}^{3}$. Actually, the above argument tells us that a general curve of this family is non-degenerate in a $\mathbf{P}^{3}$. We denote by $\Pi_{\Sigma}$ the $\mathbf{P}^{3}$ spanned by the general curve $\Sigma \in \mathcal{D}$. In this way we have a 2 -dimensional family $\mathcal{S}$ of $\mathbf{P}^{3}$ 's in $\mathbf{P}^{r}$.

By the non-degeneracy assumption on $X$ in $\mathbf{P}^{r}, r \geq 6$, two general $\mathbf{P}^{3}$ 's in $\mathcal{S}$ cannot meet along a plane. We claim that:

Claim 4.1: If two general $\mathbf{P}^{3}$ 's in $\mathcal{S}$ meet along a line, then $r=6$ and $X$ sits in a cone over the Veronese surface in $\mathbf{P}^{5}$, and claim 4 holds for $X$.

The latter assertion immediately follows from the former. In order to prove this, note that the lines which are intersections of two general $\mathbf{P}^{3}$ 's in $\mathcal{S}$ have to meet pairwise; otherwise all the $\mathbf{P}^{3}$ 's in $\mathcal{S}$ would be contained in the $\mathbf{P}^{5}$ spanned by two general $\mathbf{P}^{3}$ 's in $\mathcal{S}$, contradicting the non-degeneracy of $X$. Then either all the lines in question, hence all the $\mathbf{P}^{3}$ 's in $\mathcal{S}$, contain a fixed point $P \in \mathbf{P}^{r}$, or they all lie in a plane $Z$. The latter case cannot occur. In fact, by projecting $X$ from $Z$ to $\mathbf{P}^{r-3}$, we would find a surface $X^{\prime}$ with a 2-dimensional family of lines. Hence $X^{\prime}$ would be a plane and therefore it would be degenerate, because $r-3 \geq 3$, a contradiction again. On the other hand, by projecting $X$ to $\mathbf{P}^{r-1}$ from $P$ we find a non-degenerate surface $X^{\prime}$ with a 2-dimensional family of irreducible plane curves. As we saw, $X^{\prime}$ is a Veronese surface, proving claim 4.1.

We may thus assume, from now on, that two general $\mathbf{P}^{3}$ 's in $\mathcal{S}$ span a $\mathbf{P}^{6}$. Notice in fact that they cannot be skew, since two general curves in $\mathcal{D}$ of course meet. We want to show that this case does not really occur. 
We consider the tangential projection $\tau_{X}: X \rightarrow X_{1} \subset \mathbf{P}^{r-3}$ from a general point $P$. Since $r \geq 6$, then $r-3 \geq 3$. By proposition 3.6, the surface $X_{1}$ is 0 -defective; hence it is a developable scroll. Notice that the generating lines of $X_{1}$ are the projection of the curves of $\mathcal{D}(-P)$.

We will prove that $X_{1}$ can be neither a cone, nor the developable tangent to a curve, thus concluding the proof of claim 4 in the case $k=1$.

Claim 4.2: $X_{1}$ is not a cone.

Suppose $X_{1}$ is a cone. Then two general curves $\Sigma, \Sigma^{\prime}$ in $\mathcal{D}(-P)$ would be mapped to two lines which meet at a point, thus spanning a plane $S$. Hence $\Sigma \cup \Sigma^{\prime}$ would be contained in the $\mathbf{P}^{5}$ spanned by $T_{X, P}$ and $S$, against our assumpion that $\Pi_{\Sigma}$ and $\Pi_{\Sigma^{\prime}}$ span a $\mathbf{P}^{6}$.

Claim 4.3: $X_{1}$ is not the developable tangent to a curve.

By remark 5.11, two general curves in $\mathcal{D}(-P)$ meet transversely at $P$. By (7.2) we see that two general curves in $\mathcal{H}(-2 P)$ have nodes with different tangents at $P$, i.e. they have intersection multiplicity 4 at $P$. One moment of reflection then shows that the image of the interminacy point $P$ under the rational map $\tau_{X}$ is an irreducible curve $\Gamma$ of degree $\gamma \leq 2$, which meets all the rulings of $X_{1}$. Hence $\Gamma$ is a conic and $X_{1}$ is a rational scroll. On the other hand, the general curve $\Sigma \in \mathcal{D}$ is mapped via $\tau_{X}$ to a curve $\bar{\Sigma}$ which does not meet $\Gamma$. By letting $\Sigma$ move to a general curve in $\mathcal{D}(-P)$, we see that $\bar{\Sigma}$ is homologous, on $X_{1}$, to $\Gamma$ plus a ruling. Hence $\bar{\Sigma}$ is a cubic. Then, since $X_{1}$ is rational, we see that its degree has to be 5 ; thus $X_{1}$ cannot be the developable tangent to a curve, because such a surface has even degree.

Once we have proved claim 4 in the case $k=1$, the general case is easily proved by induction, by looking at a general tangential projection and using proposition 3.6. We leave the details to the reader.

Consider now the rational map $\phi: X \rightarrow Y \subseteq \mathbf{P}^{k+1}$ determined by $\mathcal{D}$. Notice that it is generically finite to the image $Y$, which is therefore a surface.

Since $\mathcal{H}$ contains the involution $\mathcal{D}^{(2)}$, which in turn is contained in the linear system $\mathcal{D}_{2}$ the pull-back via $\phi$ of the quadrics of $\mathbf{P}^{k+1}$, we have, by theorem 6.1 applied to $Y$ for $\iota=0$, by (7.3) and by claim 4 , that

$$
3(k+1)-\delta_{k}=r=\operatorname{dim}(\mathcal{H}) \geq \operatorname{dim}\left(\mathcal{D}_{2}\right) \geq 3 k+2,
$$

which yields $\delta_{k} \leq 1$.

Claim 5: If $X$ is $k$-defective, then $\delta_{k}=1$, and we are in case (i) of theorem 1.3.

If $\delta_{k}>0$, then, as we saw, we must have $\delta_{k}=1$. Furthermore, (7.4) tells us that $\operatorname{dim}\left(\mathcal{D}_{2}\right)=3 k+2$. Then theorem 6.1 implies that $\mathcal{D}$ is a complete linear system of dimension $k+1$ and $Y$ is a surface of minimal degree $k$ in $\mathbf{P}^{k+1}$ with rational normal sections. In addition, (7.4) implies that $\mathcal{H}=\mathcal{D}_{2}$. Since $\mathcal{H}$ is very ample on $X$, we thus deduce that $\phi$ must be an isomorphism to the image, proving the claim.

We turn now to the case of non- $k$-defective surfaces. We will dispose of this case with the next two claims. First we notice that, if $\delta_{k}=0$, then (7.4) tells us that either

$$
\operatorname{dim}\left(\mathcal{D}_{2}\right)=3 k+2
$$

or

$$
\operatorname{dim}\left(\mathcal{D}_{2}\right)=3 k+3 .
$$


Claim 6: If $X$ is not $k$-defective, i.e. $\delta_{k}=0$, and (7.5) holds, then we are in case (iv) of theorem 1.3.

Again, as before, we have that $\mathcal{D}$ is a complete linear system of dimension $k+1$ and $Y$ is a surface of minimal degree $k$ in $\mathbf{P}^{k+1}$ with rational normal sections. By (7.3) and claim 4 we have

$$
\operatorname{dim}(\mathcal{H})=r=3(k+1)=(3 k+2)+1=\operatorname{dim}\left(\mathcal{D}_{2}\right)+1 .
$$

The assertion immediately follows.

Claim 7: If $\delta_{k}=0$, and (7.6) holds, then we are either in case (iii) or in case (v) of theorem 1.3.

In the present situation we have $\mathcal{H}=\mathcal{D}_{2}$ and, as in the proof of claim 5 , we deduce that $\phi$ must be an isomorphism to the image.

Let us now apply theorem 6.1 to $Y \subseteq \mathbf{P}^{k+1}$. Let $d$ be the degree of $Y$. If $d=q+1=k$, then $Y$ would be a surface of minimal degree and $\mathcal{D}_{2}=\mathcal{H}$ would have dimension $3 k+2$, a contradiction. Thus we have $d \geq q+2=k+1$ and $q \geq 1$.

Suppose $q \geq 2$ and $d \geq q+3$. Then theorem 6.1 could be applied with $\iota=2$ and would tell us that

$$
3(k+1)=\operatorname{dim}(\mathcal{H})=\operatorname{dim}\left(\mathcal{D}_{2}\right)=h_{Y}(2)-1 \geq 3(k+1)+1,
$$

a contradiction. Thus, if $q \geq 2$, then $d=q+2=k+1$, and theorem 6.1 again tells us we are in case (v) of theorem 1.3. Otherwise $q=1$, and we are in case (iii).

Finally we analyse

Case (ii): The general divisor $\Sigma$ is reducible.

By theorem $5.10, \mathcal{D}$ is composite with a pencil $\mathcal{P}$. Then $\Sigma=\Sigma_{P_{1}, \ldots, P_{k+1}}=$ $F_{1}+\cdots+F_{k+1}$, where $F_{i}$ is the curve of the pencil through $P_{i}, i=1, \ldots, k+1$.

Claim 8: Suppose that $X$ lies in an $s+2$-dimensional cone as in (ii) or (iii) of theorem 1.3. Then:

(i) if the rulings of the cone cut out on $X$ the curves of the pencil $\mathcal{P}$, then $r \geq 2 k+s+3$

(ii) conversely, if $r \geq 2 k+s+3$, then the rulings of the cone cut out on $X$ the curves of the pencil $\mathcal{P}$.

Indeed, let $V$ be the $s$-dimenional vertex of the cone. To prove (i), let $\mathcal{H}^{\prime}$ be the linear system of hyperplane sections of $X$ through $V$. Then $\mathcal{H}^{\prime}$ is composed with the pencil $\mathcal{P}$. Moreover it contains $2 F_{1}+\cdots+2 F_{k+1}$, where $F_{1}, \ldots, F_{k+1}$ are $k+1$ general curves of the pencil. This immediately implies that

$$
r-s-1=\operatorname{dim}\left(\mathcal{H}^{\prime}\right) \geq 2(k+1),
$$

proving (i). Part (ii) immediately follows by what we saw in example 4.3.

By part (i) of the previous claim, we may, and will, replace the original $k$ by the integer $s \leq k$ such that $X$ is $s$-weakly defective, but not $(s-1)$-weakly defective. We will still assume that $s \geq 1$, since otherwise we are done.

Notice that, by our assumptions, we have

$$
\begin{gathered}
\operatorname{dim}\left(\mathcal{H}\left(-2 F_{1}-\cdots-2 F_{s}\right)\right) \leq r-3 s-1, \\
\operatorname{dim}\left(\mathcal{H}\left(-2 F_{1}-\cdots-2 F_{s+1}\right)\right) \geq r-3(s+1)+\delta_{s} .
\end{gathered}
$$

Hence

$$
2 \leq \operatorname{dim}\left(\mathcal{H}\left(-2 F_{1}-\cdots-2 F_{s}\right)\right)-\operatorname{dim}\left(\mathcal{H}\left(-2 F_{1}-\cdots-2 F_{s+1}\right)\right) \leq 2-\delta_{s}
$$

and therefore $\delta_{s}=0$ and the equality holds everywhere. In particular, we conclude that: 
Claim 9: In the present situation, we have:

(i) $r=3(s+1)$;

(ii) the general curve $F$ in $\mathcal{P}$ is contracted to a point by the rational map determined by the linear system $\mathcal{H}\left(-2 F_{1}-\cdots-2 F_{s}\right)$; and

(iii) $X$ is not $s$-defective, but it is l-defective for all $l \geq s+1$ for which $\mathcal{H}\left(-2 F_{1}-\cdots-2 F_{s+1}\right)$ is not empty.

The first assertion follows by (7.7). Part (ii) easily follows from the fact that if $F$ is the general curve in the pencil $\mathcal{P}$, then $2 F$ imposes exactly two conditions on $\mathcal{H}\left(-2 F_{1}-\cdots-2 F_{s}\right)$, so that $F$ has to impose one condition on the same system. For the same reason, $2 F$ imposes two conditions on the systems $\mathcal{H}\left(-2 F_{1}-\cdots-2 F_{l}\right)$, as soon as $l \geq k+1$ and $\mathcal{H}\left(-2 F_{1}-\cdots-2 F_{l+1}\right)$ is not empty. This implies (iii).

Now theorem 1.3 will be proved if we prove that we are in case (vi), which we will do next.

Recall that by theorem 1.1 we have $h(\Sigma)=h\left(F_{1}+\cdots+F_{s+1}\right) \leq 2 s+2$. On the other hand, $h\left(F_{1}+\cdots+F_{s+1}\right) \geq h\left(F_{1}\right)+s$; hence $h\left(F_{1}\right) \leq s+2$. In other words, a general curve $F$ of the pencil $\mathcal{P}$ spans a projective space $\Pi_{F}$ of dimension $h \leq s+1$. By part (ii) of claim 9, for a general $F \in \mathcal{P}$, the space $\Pi_{F}$ meets a subspace of dimension $3 k$ along a subspace of dimension $h-1$. Let $V$ be a subspace of minimal dimension $v$ which is cut by the general $\Pi_{F}$ along a subspace of dimension $h-1$. Then $X$ sits in a $(v+2)$-dimensional cone over a curve with vertex $V$ and, according to example 4.3 and claim 8 , the theorem will be proved if we prove that $v \leq s$. In this case, we note that $V$ has dimension $s$ by example 4.3 and is uniquely determined. Otherwise $X$ would be contained in the span of two such spaces, which is a proper subspace in $\mathbf{P}^{3(s+1)}$.

First we dispose of the case $s=1$.

Claim 10: Theorem 1.3 holds if $s=1$.

In this case $r=6$, and $F$ is either a non-degenerate plane curve or a line.

If $F$ spans a plane, then $X$ is swept out by plane curves, whose planes pairwise meet along a line. Then either all these planes span a $\mathbf{P}^{3}$ which contains $X$, a contradiction, or they all contain the same line, and we are in case (vi) of theorem 1.3 .

If $F$ is a line, then $X$ is a scroll, which is not developable. Let, as above, $F_{1}$, $F_{2}$ be two general rulings of $X$ and let $S_{1}$ and $S_{2}$ be the tangent $\mathbf{P}^{3}$ 's along $F_{1}$ and $F_{2}$, i.e. the $\mathbf{P}^{3}$ 's spanned by the tangent planes to $X$ along $F_{1}$ and $F_{2}$. In the present situation, $S_{1} \cup S_{2}$ spans the same $\mathbf{P}^{5}$ spanned by the tangent planes to $X$ at two general points, one on $F_{1}$, one on $F_{2}$. This means that the general tangent plane to $X$ meets $S_{1}$ at a point, which, by proposition (4.1), implies that by projecting $X$ down to $\mathbf{P}^{r-4}$ from $S_{1}$ we get a curve $\Gamma$. Since $r \geq 6, \Gamma$ cannot be a line. Hence the aforementioned projection contracts all the rulings of $X$ to points of $\Gamma$. In other words, the rulings in question all meet $S_{1}$. Since they also meet $S_{2}$, but they cannot lie in a fixed $\mathbf{P}^{5}$, then they all meet the line $S_{1} \cap S_{2}$, and we are again in case (vi) of theorem 1.3 .

Next we turn to the case $s \geq 2$, and proceed by induction on $s$. Since $r=3(s+1)$, we may apply proposition 3.6 to $X$. So we make a general tangential projection of $X$ and get an $(s-1)$-defective surface $X_{1}$ in $\mathbf{P}^{r^{\prime}}$, with $r^{\prime} \geq 3 s$, to which we can apply induction. Hence $X_{1}$ sits in a cone over a curve with vertex $V_{1}$, a subspace of dimension $s-1$ and not less.

By proposition (4.1) we know that $V$ is cut by the general tangent plane to $X$ in a point. On the other hand, we have 
Claim 11: Let $F$ be a general curve in $\mathcal{P}$. Then either $\Pi_{F}$ does not intersect the general tangent plane of $X$, or $v \leq s$.

Suppose $\Pi_{F}$ intersects the general tangent plane of $X$. If $F_{1}, F_{2}$ are two general curves in $\mathcal{P}$, then either the general tangent plane to $X$ intersects $\left\langle\Pi_{F_{1}}, \Pi_{F_{2}}\right\rangle$ along a line or it intersects $\Pi_{F_{1}}, \Pi_{F_{2}}$ along their intersection. But $\left\langle\Pi_{F_{1}}, \Pi_{F_{2}}\right\rangle$ has dimension at most $2 s+3 \leq 3 s+1$, because $s \geq 2$. Thus, by proposition 4.1, only the second possibility holds. But then the general tangent plane to $X$ has to intersect the intersection $V^{\prime}$ of all the $\Pi_{F}$ 's. Notice that $V^{\prime}$ has dimension at most $s$. Furthermore, by proposition 4.1 again, $X$ sits in a cone over a curve with vertex $V^{\prime}$. By part (ii) of claim 8, we see that the rulings of the cone cut out on $X$ the curves of $\mathcal{P}$, which implies that, for a general $F$, the space $\Pi_{F}$ cuts $V^{\prime}$ in a subspace of dimension $h-1$. This proves our assertion.

So we may assume that for $F$ general in $\mathcal{P}, \Pi_{F}$ does not intersect the general tangent plane of $X$. Going back to the tangential projection of $X$ to $X_{1}$ from a general tangent plane $T_{X, P}$ of $X$, we have the subspace $W_{P}:=\left\langle T_{X, P}, V_{1}\right\rangle$ of dimension $s+2$. A general $\Pi_{F}$ is projected to a subspace of the same dimension $h$, intersecting $V_{1}$ in a subspace of dimension $h-1$. In particular, $h \leq s$, and $\Pi_{F}$ has to intersect $W_{P}$ in a subspace of dimension $h-1$. This shows that $v \leq s+2$.

Finally we can conclude our proof that $v=s$. Notice that $X_{1}$ is swept out by curves $F^{\prime}$, the projections of the curves $F$ in $\mathcal{P}$, spanning subspaces $\Pi_{F^{\prime}}$, the projections of the $\Pi_{F}$ 's, which, as we saw, have dimension $h$ and cut $V_{1}$ in a subspace of dimension $h-1$. On the other hand, a subspace $V$ would project down to a subspace $V^{\prime}$ which is also met by the $\Pi_{F^{\prime}}$ 's in a subspace of dimension $h-1$. Since $V$ is met by the general tangent plane of $X$ at a point, then $\operatorname{dim}\left(V^{\prime}\right)=v-1 \leq s+1$. Thus the span of $V^{\prime}$ and $V_{1}$ would have dimension at most $2 s+1<3 s$. This implies that the general space $\Pi_{F^{\prime}}$ meets $V_{1} \cap V^{\prime}$; otherwise $\left\langle V_{1}, V^{\prime}\right\rangle$ would contain the general $\Pi_{F^{\prime}}$, hence the whole of $X_{1}$, a contradiction. Since there is no subspace of $\mathbf{P}^{3 s}$ of dimension smaller than $s-1$ which is met by the general $\Pi_{F^{\prime}}$ in a subspace of dimension $h-1$, we have that $V^{\prime}$ has to contain $V_{1}$. Hence $V \cap W_{P}$ has dimension $s$, and therefore $\left\langle V, W_{P}\right\rangle$ has dimension at most $s+4<3(s+1)$. Since the general $\Pi_{F}$ meets both $V$ and $W_{P}$ in a subspace of dimension $h-1$, and cannot be contained in $\left\langle V, W_{P}\right\rangle$, then it has to intersect $V \cap W_{P}$ in a subspace of dimension $h-1$, which tells us, by the minimality property of $V$, that $V \subset W_{P}$; hence it has dimension $s$.

\section{REFERENCES}

[Adl] Adlandsvik B., Joins and Higher secant varieties, Math. Scand. 61 (1987), 213-222. MR 89j:14030

[ArCo] Arbarello E., Cornalba M., Footnotes to a paper of B. Segre, Math. Ann. 256 (1981), 341-362. MR 83d:14016

[Br] Bronowski J., Surfaces whose prime sections are hyperelliptic, J. London Math. Soc. 8 (1933), 308-312.

[CJ1] Catalano-Johnson M., The possible dimensions of the higher secant varieties, Amer. J. Math. 118 (1996), 355-361. MR 97a:14058

[CJ2] Catalano-Johnson M., When do $k$ general double points impose independent conditions on degree d plane curves?, Curves Seminar of Queen's, Vol. X, Queen's Pap. Pure Appl. Math. 102, Queen's Univ., Kingston, Ont., 1995, pp. 166-181. MR 97g:14038

[ChCi] Chiantini L., Ciliberto C., A few remarks on the lifting problem, Asterisque 218 (1993), 95-109. MR 95c:14072

[CiHi] Ciliberto C., Hirschowitz A., Hypercubiques de $\mathbf{P}^{4}$ avec sept pointes singulieres generiques, C. R. Acad. Sci. Paris Sér. I Math. 313 (1991), 135-137. MR 92g:14043 
[CiLoMi] Ciliberto C., Lopez A., Miranda R., Some remarks on the obstructedness of cones over curves of low genus, in Higher dimensional complex varieties, Proceedings Trento 1994, De Gruyter (1996), 167-182. MR 98i:14006

[Ci] Ciliberto C., Hilbert functions of finite sets and the genus of a curve in projective space, Springer Lect. Notes in Math. 1266 (1987), 24-73. MR 89c:14039

[CiSe] Ciliberto C., Sernesi E., Singularities of the theta divisor and congruences of planes, J. Alg. Geom. 1 (1992), 231-250. MR 92j:14034

[Dale1] Dale M., Terracini's lemma and the secant variety of a curve, Proc. London Math. Soc. (3) 49 (1984), 329-339. MR 85g:14066

[Dale2] Dale M., On the secant variety of an algebrac surface, University of Bergen, Dept. of Math. preprint no. 33 (1984).

[DiG] Di Gennaro V., Self intersection of the canonical bundle of a projective variety, to appear in Comm. in Alg..

[EiHa] Eisenbud D., Harris J., Curves in projective spaces, Montreal University Press (1982). MR 84g:14024

[EnChi] Enriques F., Chisini O., Teoria geometrica delle equazioni e delle funzioni algebriche, vol. III, Zanichelli (Bologna) (1985). MR 90b:01106b

[Fa] Fantechi, B., On the superadditivity of secants defects, Bull. Soc. Math. France 118 (1990), 85-100. MR 92c:14049

[Ga] Gallarati D., Alcune osservazioni sopra le varietà $i$ cui spazi tangenti si appoggiano irregolarmente a spazi assegnati, Rend. Accad. Naz. Lincei, VIII 20 (1956), 193-199. MR 18:231b

[GrHa] Griffiths Ph., Harris J., Algebraic geometry and local differential geometry, Ann. Scient. Ec. Norm. Sup. 12 (1979), 335-432. MR 81k:53004

[Ha] Harris J., A bound on the geometric genus of projective varieties, Ann. Scuola Norm. Sup. Pisa Cl. Sci. (4) 8 (1981), 35-68. MR 82h:14010

[Ma] Matsusaka T., On a theorem of Torelli, Amer. J. of Math. 80 (1958), 801-821. MR 20:3867

[Pal1] Palatini F., Sulle varietà algebriche per le quali sono di dimensione minore dell'ordinario, senza riempire lo spazio ambiente, una o alcune delle varietà formate da spazi seganti, Atti. Accad. Torino 44 (1909), 362-374.

[Pal2] Palatini F., Sulle superficie algebriche $i$ cui $S_{h}(h+1)$-seganti non riempiono lo spazio ambiente, Atti. Accad. Torino 41 (1906), 634-640.

[Sco1] Scorza G., Determinazione delle varietá a tre dimensioni di $S-r, r \geq 7, i$ cui $S_{3}$ tangenti si tagliano a due a due., Rend. Circ. Mat. Palermo 25 (1908), 193-204.

[Sco2] Scorza G., Un problema sui sistemi lineari di curve appartenenti a una superficie algebrica, Rend. R. Ist. Lombardo (2) 41 (1908), 913-920.

[Segre] Segre C., Preliminari di una teoria delle varietá luoghi di spazi, Rend. Circ. Mat. Palermo 30 (1910), 87-121.

[Terr1] Terracini A., Sulle $V_{k}$ per cui la varietà degli $S_{h}(h+1)$-seganti ha dimensione minore dell' ordinario, Rend. Circ. Mat. Palermo 31 (1911), 392-396.

[Terr2] Terracini A., Su due problemi, concernenti la determinazione di alcune classi di superficie, considerati da G. Scorza e F. Palatini, Atti Soc. Natur. e Matem. Modena (V) 6 (1921-22), 3-16.

[Zak] Zak F., Tangents and secants of algebraic varieties, Transl. Math. Monogr. 127, Amer. Math. Soc., Providence, RI, (1993). MR 94i:14053

Department of Mathematics, University of Siena, Via del Capitano 15, 53100 Siena, ITALY

E-mail address: chiantini@unisi.it

Department of Mathematics, University of Rome II, Viale della Ricerca Scientifica, 16132 Rome, ItALY

E-mail address: cilibert@axp.mat.uniroma2.it 